\title{
Hepatitis C Virus Infection Is Inhibited by a Noncanonical Antiviral Signaling Pathway Targeted by NS3-NS4A
}

\author{
Christine Vazquez, ${ }^{a}$ Chin Yee Tan, ${ }^{a}$ (D) Stacy M. Hornera,b \\ aDepartment of Molecular Genetics and Microbiology, Duke University Medical Center, Durham, North Carolina, USA \\ bDepartment of Medicine, Duke University Medical Center, Durham, North Carolina, USA
}

ABSTRACT The hepatitis C virus (HCV) NS3-NS4A protease complex is required for viral replication and is the major viral innate immune evasion factor. NS3-NS4A evades antiviral innate immunity by inactivating several proteins, including MAVS, the signaling adaptor for RIG-I and MDA5, and Riplet, an E3 ubiquitin ligase that activates RIG-I. Here, we identified a Tyr-16-Phe (Y16F) change in the NS4A transmembrane domain that prevents NS3-NS4A targeting of Riplet but not MAVS. This Y16F substitution reduces HCV replication in Huh7 cells, but not in Huh-7.5 cells, known to lack RIG-I signaling. Surprisingly, deletion of RIG-I in Huh7 cells did not restore Y16F viral replication. Rather, we found that Huh-7.5 cells lack Riplet expression and that the addition of Riplet to these cells reduced HCV Y16F replication, whereas the addition of Riplet lacking the RING domain restored HCV Y16F replication. In addition, TBK1 inhibition or IRF3 deletion in Huh7 cells was sufficient to restore HCV Y16F replication, and the Y16F protease lacked the ability to prevent IRF3 activation or interferon induction. Taken together, these data reveal that the NS4A Y16 residue regulates a noncanonical Riplet-TBK1-IRF3-dependent, but RIG-I-MAVS-independent, signaling pathway that limits HCV infection.

IMPORTANCE The HCV NS3-NS4A protease complex facilitates viral replication by cleaving and inactivating the antiviral innate immune signaling proteins MAVS and Riplet, which are essential for RIG-I activation. NS3-NS4A therefore prevents IRF3 activation and interferon induction during $\mathrm{HCV}$ infection. Here, we uncover an amino acid residue within the NS4A transmembrane domain that is essential for inactivation of Riplet but does not affect MAVS cleavage by NS3-NS4A. Our study reveals that Riplet is involved in a RIG-I- and MAVS-independent signaling pathway that activates IRF3 and that this pathway is normally inactivated by NS3-NS4A during HCV infection. Our study selectively uncouples these distinct regulatory mechanisms within NS3-NS4A and defines a new role for Riplet in the antiviral response to HCV. Since Riplet is known to be inhibited by other RNA viruses, such as such influenza A virus, this innate immune signaling pathway may also be important in controlling other RNA virus infections.

KEYWORDS HCV, MAVS, NS3-NS4A, Riplet, antiviral innate immunity, immune evasion, protease

H epatitis C virus (HCV) is a positive-sense, single-stranded RNA virus that infects over 70 million people worldwide, with up to $80 \%$ of infected individuals developing chronic infection (1). The recent development of direct-acting antivirals for HCV has dramatically improved successful treatment of HCV infection (2). However, many HCV-infected individuals are asymptomatic and thus unaware of their HCV status until secondary manifestations, such as liver cirrhosis and hepatocellular carcinoma, arise decades later. Notably, although the current direct-acting antivirals treat HCV-induced disease, they do not always prevent reinfection in cured individuals. Therefore, there is

Citation Vazquez C, Tan CY, Horner SM. 2019 Hepatitis C virus infection is inhibited by a noncanonical antiviral signaling pathway targeted by NS3-NS4A. J Virol 93:e00725-19. https://doi.org/10.1128/JVI.00725-19. Editor J.-H. James Ou, University of Southern California

Copyright $\odot 2019$ American Society for Microbiology. All Rights Reserved.

Address correspondence to Stacy M. Horner, stacy.horner@duke.edu.

Received 1 May 2019

Accepted 16 September 2019

Accepted manuscript posted online 18

September 2019

Published 13 November 2019 
an urgent need for future studies into the development of a vaccine to reduce the global burden of HCV infection (3).

Several factors contribute to the ability of HCV to establish a chronic infection, including its ability to evade detection and dysregulate the host antiviral innate immune response through the actions of the HCV NS3-NS4A protease complex (4). The NS3-NS4A protease is a protein complex formed between NS3, which contains protease and helicase domains, and NS4A. NS4A is a 54-amino-acid protein that contains an $\mathrm{N}$-terminal transmembrane domain, an NS3 interacting domain, and a C-terminal acidic domain (5). The NS4A transmembrane domain anchors NS3 to membranes (6) and mediates NS4A dimerization (7). NS3-NS4A has diverse functions in the HCV life cycle, with roles in HCV RNA replication, viral assembly, and innate immune evasion (reviewed in references 8 and 9). The mechanisms that regulate these diverse functions of NS3-NS4A are not completely understood. However, it is known that NS4A directs the protease complex to distinct intracellular membranes to perform some of these functions: the endoplasmic reticulum (ER) for viral replication and mitochondria and mitochondrial-ER contact sites (often referred to as mitochondrial-associated ER membranes [MAM]) for immune evasion (10-14).

Antiviral innate immune signaling against HCV can be initiated by the RNA sensor proteins RIG-I and MDA5 (15-17). RIG-I is directly activated by multiple ubiquitination events by E3 ubiquitin ligases, namely, TRIM25 and Riplet, which binds to and adds K63-linked ubiquitin chains to RIG-I but not MDA5 (18-21). Once activated, RIG-I and MDA5 signal to the adaptor protein MAVS to drive a signal transduction cascade that induces the phosphorylation of IRF3 and then the transcriptional induction of beta interferon (IFN- $\beta$ ). HCV infection can also be sensed by TLR3, which signals via TRIF and IRF3 to induce antiviral innate immunity (22). During HCV infection, NS3-NS4A cleaves and/or inactivates MAVS $(10,12,23,24)$, TRIF (25), and Riplet (19) to block IRF3 activation (26).

Here, we aimed to uncouple the roles of NS3-NS4A in replication and immune evasion. We focused on the NS4A transmembrane domain and found a residue, Y16, that regulates inactivation of Riplet, but not MAVS, revealing a new branch of innate immune signaling that controls HCV infection.

(This article was submitted to an online preprint archive [27].)

\section{RESULTS}

A Y16F substitution in NS4A disrupts replication of an HCV subgenomic replicon in Huh7 cells, but not in Huh-7.5 cells. The transmembrane domain of NS4A contains two aromatic amino acids: a tryptophan at position 3 (W3) and a tyrosine at position 16 (Y16) (Fig. 1A). These two aromatic amino acids, which are conserved in all sequenced HCV strains in the Los Alamos HCV sequence database (28) (Fig. 1A), are located at each end of the NS4A transmembrane domain at the lipid bilayer interface $(5,7)$. Interestingly, aromatic residues at the termini of transmembrane domains are often important for positioning membrane proteins within lipid bilayers (29-31). Therefore, we hypothesized that these residues may play a role in the proper localization and/or function of the NS3-NS4A protease complex during HCV infection. While both the $\mathrm{W} 3$ and the $\mathrm{Y} 16$ residues in NS4A are conserved across the eight known HCV genotypes, we chose to focus specifically on the $\mathrm{Y} 16$ residue (Fig. 1A), with the goal of uncoupling the function of Y16 in HCV replication from targeting of innate immune substrates, such as MAVS and Riplet. Since a prior study found that a Y16A substitution inhibited HCV replication (7), we made the more conservative phenylalanine mutation (Y16F) to maintain aromaticity at this position. Here, we analyzed the role of this amino acid in regulating HCV replication and innate immune regulation by NS3-NS4A.

To determine whether the Y16F substitution in NS4A altered HCV replication, we first engineered this amino acid change into an HCV replicon encoding a G418 marker (HCV genotype 1B subgenomic replicon; HP replicon [15]). After in vitro transcription, wild-type (WT) or Y16F HCV replicon RNA was electroporated into either liver hepatoma Huh-7.5 cells, which do not have functional RIG-I signaling due to the T55I mutation 


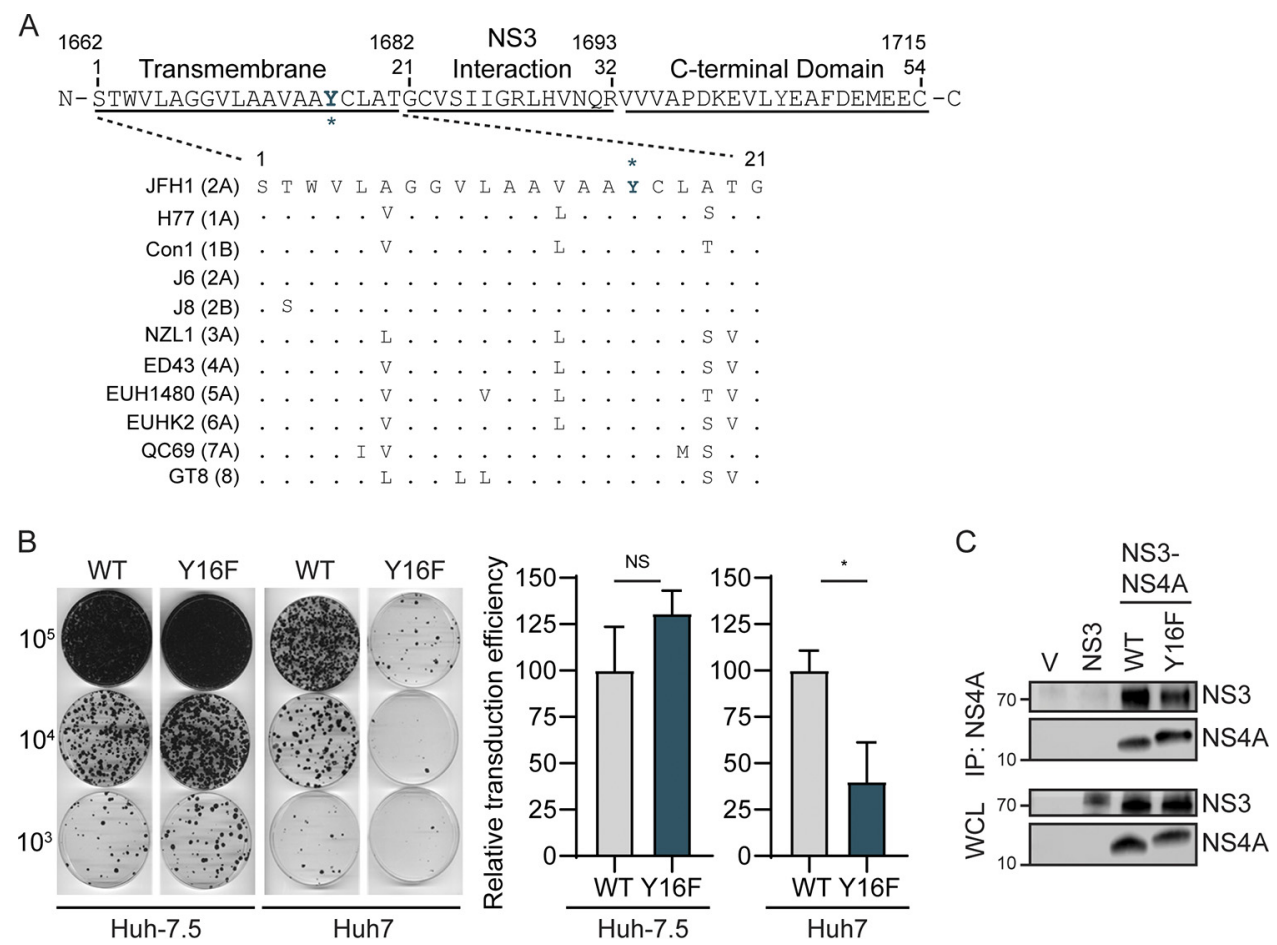

FIG 1 A Y16F substitution in NS4A disrupts replication of an HCV subgenomic replicon in Huh7 cells, but not in Huh-7.5 cells. (A) Amino acid sequence of NS4A, with the Y16 residue starred and indicated in teal. Numbers correspond with the amino acid position within NS4A (amino acids 1 to 54) or the full-length HCV polyprotein (amino acids 1662 to 1715). Strain names are listed as found in the Los Alamos HCV sequence database. Conserved amino acids are indicated with a dot, whereas differences are listed. (B) Representative images of Huh-7.5 or Huh7 cells electroporated with the in vitro-transcribed HCV subgenomic replicon RNA (HP, genotype 1B; WT or Y16F). Cells were plated in serial dilutions $\left(2 \times 10^{5}, 2 \times 10^{4}\right.$, and $\left.2 \times 10^{3}\right)$ and then stained with crystal violet after 3 weeks of G418 selection. Graphs show the relative transduction efficiency, which denote the percentages of colonies in Y16F-transduced cells relative to the WT. Bars indicate means \pm the SEM ( $n=$ three to four biological replicates), with data analyzed by Student $t$ test $\left({ }^{*}, P<0.05\right.$; NS, not significant). (C) Immunoblot analysis of anti-NS4A immunoprecipitated extracts or whole-cell lysate (WCL) from 293T cells transfected with the indicated HCV proteins (genotype 1B) or vector (V). Panels are representative of three independent experiments.

(15), or Huh7 cells, which have functional RIG-I signaling. In the Huh-7.5 cells, the number of G418-resistant colonies in the WT versus the Y16F HCV replicon-transduced cells was equivalent, indicating that WT and Y16F replicated similarly. However, in Huh7 cells, the Y16F HCV replicon had a reduced transduction efficiency ( $\sim$-fold) compared to the WT HCV replicon (Fig. 1B). As a control, we also measured the interaction of NS4A WT or Y16F with NS3 by coimmunoprecipitation and found that the Y16F substitution did not alter the interaction of NS4A with NS3 or the ability of the NS3-NS4A protease to process the NS3-NS4A polyprotein junction (Fig. 1C). Together, these data reveal that the $\mathrm{Y} 16 \mathrm{~F}$ mutation results in reduced HCV replication in Huh7 cells, but not Huh-7.5 cells, suggesting that NS4A Y16F may regulate RIG-I-mediated innate immune signaling to promote HCV immune evasion and replication.

RIG-I deletion in Huh7 cells does not restore HCV NS4A Y16F viral replication. To determine whether the Y16F substitution in NS4A specifically altered HCV replication in Huh7 cells during infection, we engineered the NS4A Y16F substitution into the full-length HCV infectious clone (JFH1, genotype 2A [32]). We generated low-passagenumber viral stocks and confirmed that the $\mathrm{Y} 16 \mathrm{~F}$ mutation was maintained in the resulting virus by PCR amplification of the NS4A region and Sanger sequencing. We then infected Huh-7.5 or Huh7 cells with the HCV WT or Y16F virus, harvested protein lysates over a time course of infection, and measured HCV NS5A protein expression by immunoblotting. We found that HCV NS5A protein levels were equivalent in Huh-7.5 cells infected with WT or Y16F HCV (Fig. 2A). However, in Huh7 cells, the level of NS5A protein from the $\mathrm{Y} 16 \mathrm{~F}$ virus was reduced compared to WT HCV (Fig. 2B). To determine 
A

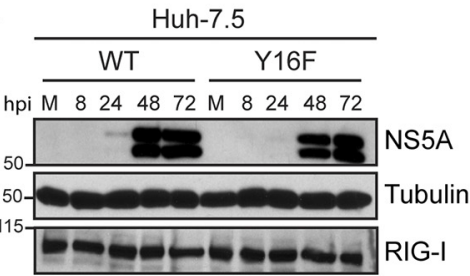

B

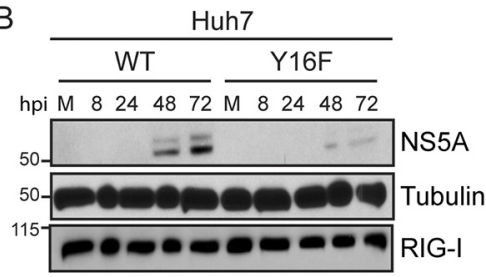

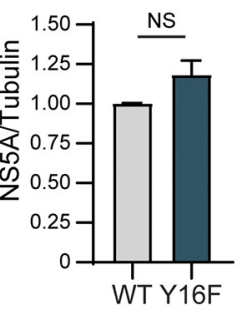

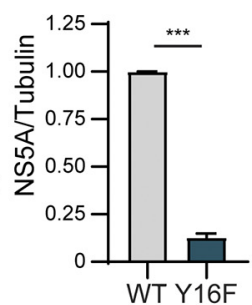

G

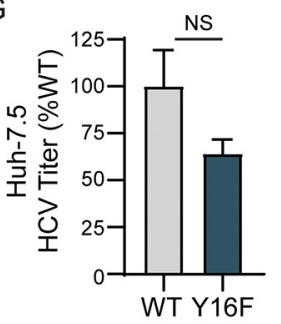

$\mathrm{H}$

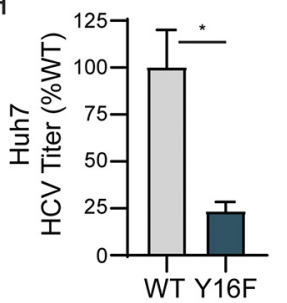

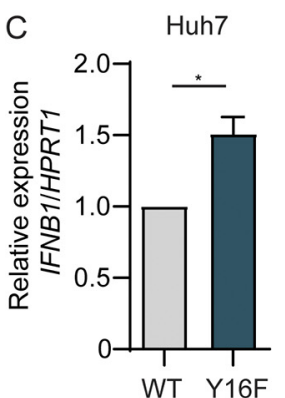

$\mathrm{F}$

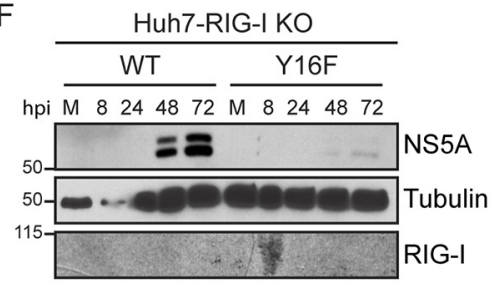

Huh7-

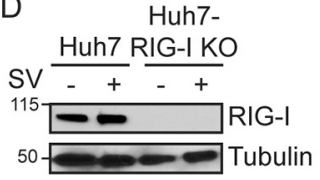

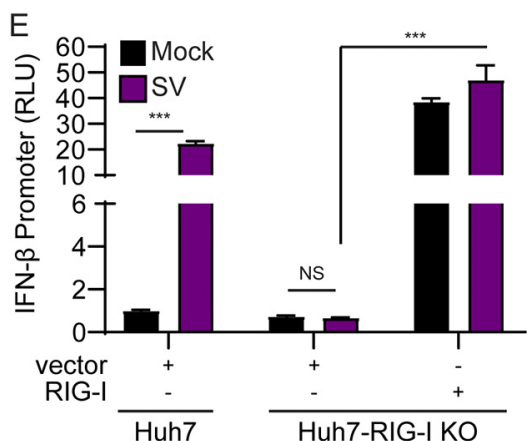

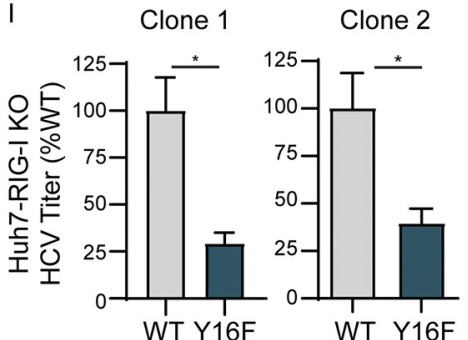

FIG 2 RIG-I deletion in Huh7 cells does not restore HCV NS4A Y16F replication. Huh-7.5 cells (A) or Huh7 cells (B) were infected with HCV, WT, or NS4A Y16F (JFH1, MOI of 0.3). Immunoblot analysis was performed on lysates extracted at the indicated hours postinfection (hpi) or after mock infection (M). Graphs directly to the right of each blot (here and in panel F) show quantification of NS5A protein relative to tubulin at 72 hpi (mean \pm the SEM; $n=$ three biological replicates). (C) IFNB1 expression relative to HPRT1 from Huh7 cells infected with HCV, WT, or NS4A Y16F (JFH1, MOI of 1 ) at $72 \mathrm{hpi}$ as analyzed by RT-qPCR, with data displayed as means \pm the SEM ( $n=$ three biological replicates). (D) Immunoblot of extracts of Huh7 and Huh7-RIG-I KO cells that were mock or SV infected (20 h). (E) IFN- $\beta$ promoter reporter luciferase expression of Huh7 and Huh7-RIG-I KO cells expressing either vector or full-length RIG-I that were either mock or SV infected $(20 \mathrm{~h})$. Values show means \pm the SD $(n=$ three technical replicates) in RLU. (F) Huh7-RIG-I KO cells were infected with HCV, WT, or NS4A Y16F (JFH1, MOI of 0.3). Immunoblot analysis was performed on lysates extracted at the indicated times or mock infected $(M)$. The graph directly to the right of this blot shows the quantification of NS5A protein relative to tubulin at $72 \mathrm{hpi}$ (mean \pm the SEM; $n=3$ biological replicates). (G to I) Focus-forming assay of supernatants harvested from Huh-7.5 (G), Huh7 (H), and Huh7-RIG-I KO (clones 1 and 2) (I) cells at $72 \mathrm{hpi} \mathrm{(MOI} \mathrm{of} \mathrm{0.3).} \mathrm{The} \mathrm{data} \mathrm{are} \mathrm{presented} \mathrm{as} \mathrm{the} \mathrm{percent} \mathrm{HCV} \mathrm{titer} \mathrm{from}$ the Y16F strain relative to the WT (set at 100\%) and show means \pm the SEM ( $n=$ two or three biological replicates), Data were analyzed by Student $t$ test $\left({ }^{*}, P<0.05 ;{ }^{* *}, P<0.01 ;{ }^{* *}, P<0.005\right.$; NS, not significant).

whether this reduction in Y16F virus replication was due to an inability to block the innate immune response, we examined induction of IFNBI mRNA by quantitative reverse transcription-PCR (RT-qPCR) during infection with both WT and Y16F viruses and found that $\mathrm{Y} 16 \mathrm{~F}$ virus was unable to block induction of IFNB1 mRNA as well as WT 
virus (Fig. 2C). In addition to RIG-I, there are likely other genetic differences between Huh7 and Huh-7.5 cells. Thus, to determine whether RIG-I was the factor accounting for the differential replication observed between WT and Y16F HCV in Huh7 cells versus Huh-7.5 cells, we generated Huh7-RIG-I KO cells using CRISPR/Cas9 genome editing. These Huh7-RIG-I KO cells contain a 252-nucleotide deletion that removes the start codon, preventing RIG-I protein expression (Fig. 2D). To confirm a loss of RIG-I signaling, we infected Huh7-RIG-I KO cells with Sendai virus (SV), a virus known to activate RIG-I signaling $(15,33)$, and observed no SV-mediated induction of RIG-I protein or signaling to the IFN- $\beta$ promoter, which was restored upon overexpression of RIG-I $(15,16)$ (Fig. 2E).

We next infected these Huh7-RIG-I KO cells with either WT or Y16F HCV and measured HCV NS5A expression from lysates harvested over a time course of infection by immunoblotting. Surprisingly, we found that NS5A protein level from Y16F HCV was not restored to the level of WT in the Huh7-RIG-I KO cells (Fig. 2F). We then compared the production of infectious virus from the WT and Y16F viruses in each of these cell lines. In these assays, the supernatants of infected cells were used to infect naive Huh-7.5 cells to determine the viral titer, which ultimately measures a second round of infection. We found that while the Y16F virus harvested from Huh-7.5 cells resulted in a somewhat lower level of infectious virus than the WT ( $40 \%$ lower), its level of infectious virus harvested from Huh7 or two independent clones of Huh7-RIG-I KO cells was significantly lower compared to WT (now $\sim 75 \%$ lower) (Fig. $2 \mathrm{G}$ to I). Taken together, these data suggest that NS4A Y16 regulates a RIG-I-independent signaling pathway that is nonfunctional in Huh-7.5 cells.

HCV NS3-NS4A Y16F retains the ability to cleave MAVS. Since NS4A Y16 is located at the membrane lipid bilayer interface $(5,7)$, and NS4A membrane interactions regulate the molecular mechanisms by which the NS3-NS4A protease targets substrates (7), we hypothesized that the Y16F substitution in NS4A may regulate NS3-NS4A cleavage of MAVS. To test this, we coexpressed NS3-NS4A with Flag-tagged MAVS and found that both NS3-NS4A WT and Y16F cleaved MAVS, while NS3-NS4A containing a mutation that inactivates the protease active site (S139A; SA) did not (Fig. 3A). We also found that MAVS cleavage was similar after HCV WT and Y16F infection in both Huh-7.5 and Huh7 cells (Fig. 3B). Further, in Huh7 cells that were either deleted of MAVS via CRISPR/Cas9 (Fig. 3B) or depleted of MAVS via shRNA (Fig. 3C), we found that Y16F viral replication was not restored to WT levels in either Huh7-MAVS KO cells, as measured by NS5A expression, or Huh7-shMAVS cells, as measured by the relative HCV copy number. Together, these findings show that MAVS does not differentially regulate HCV WT or Y16F replication and that the NS4A Y16F substitution does not alter MAVS cleavage by NS3-NS4A.

IRF3 deletion in Huh7 cells restores HCV Y16F replication to the level of HCV WT. We next wanted to determine whether the signaling pathway that inhibits HCV Y16F replication requires the IFN- $\beta$ transcription factor IRF3 (reviewed in reference 34). We first generated Huh7-IRF3 KO cells using CRISPR/Cas9 genome editing and determined the IRF3 expression and function in these cells by sequencing the IRF3 genetic locus, analyzing IRF3 expression by immunoblotting, and confirming that the loss of IRF3 prevented SV-mediated antiviral signaling to the IFN- $\beta$ promoter and that this signaling was restored by IRF3 overexpression (Fig. 4A and B). To determine whether IRF3 regulates HCV Y16F replication, we infected Huh7 or Huh7-IRF3 KO cells (two independent clones) with either HCV WT or Y16F, measured HCV NS5A expression by immunoblotting, and measured release of infectious virus by focus forming assay. Although the levels of NS5A expression and infectious Y16F virus were reduced relative to the WT in parental Huh7 cells, as before, these levels were restored to that of WT virus in two independent clones of Huh7-IRF3 KO cells (Fig. 4C and D). Together, these data reveal that NS4A Y16 regulates an IRF3-dependent signaling pathway that can inhibit HCV replication. 
A

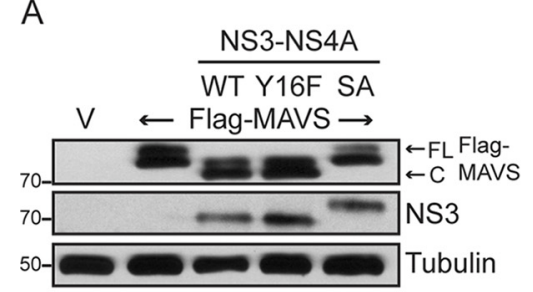

B
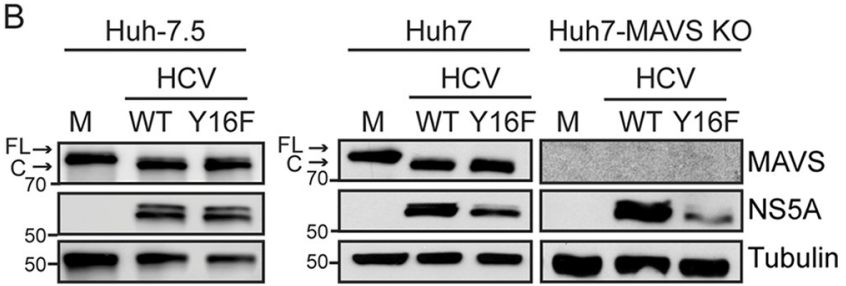

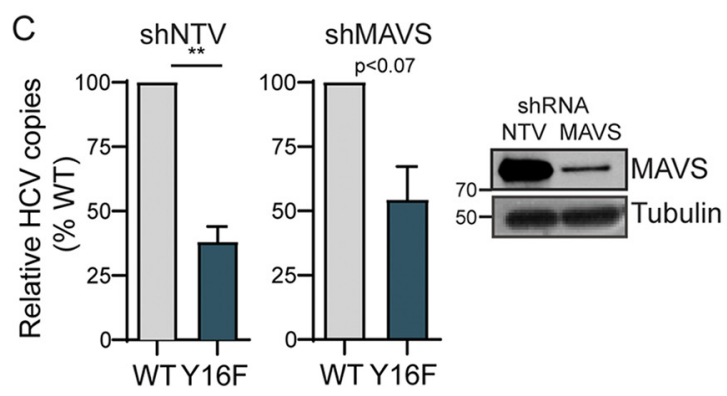

FIG 3 HCV NS3-NS4A Y16F retains the ability to cleave MAVS. (A) Immunoblot analysis of lysates harvested from Huh7 cells expressing NS3-NS4A (WT, Y16F, or SA [NS3 active-site mutant S139A]) and vector (V) or Flag-MAVS. Arrows indicate the full-length (FL) and cleaved (C) forms of MAVS. (B) Immunoblot analysis of lysates harvested at 72 hpi from Huh-7.5, Huh7, or Huh7-MAVS KO cells that were either mock infected (M) or infected with HCV, WT, or NS4A Y16F (JFH1, MOI of 0.3). Arrows indicate the full-length (FL) and cleaved (C) forms of MAVS. (C) Intracellular HCV RNA levels in Huh7 cells expressing nontargeting shRNA (shNTV) or MAVS shRNA (shMAVS) infected with HCV, WT, or NS4A Y16F (JFH1, MOI of 0.3) and harvested at $72 \mathrm{hpi}$, as measured by RT-qPCR. The data are presented as relative HCV copies from Y16F relative to WT (set at 100\%) and show the means \pm the SEM ( $n=$ two biological replicates). Immunoblots to the right of the graph shows MAVS protein expression in the shNTV and shMAVS cell lines. Data were analyzed by Student $t$ test ${ }^{* *}, P<0.01$ or as indicated).

HCV NS3-NS4A Y16F does not block IRF3 activation. Since our data suggested that HCV Y16F replication was inhibited by IRF3-mediated signaling, we hypothesized that NS3-NS4A Y16F would be unable to block IRF3 activation. During viral infection, IRF3 is activated by a multistep process, including phosphorylation by the kinases TBK1 and $\mathrm{IKK} \varepsilon$, resulting in dimerization, and finally translocation from the cytosol to the nucleus, where it activates transcription of IFN- $\beta$ (35). Importantly, it is well-known that overexpression of the WT NS3-NS4A protease can block this nuclear translocation of IRF3 in response to virus infection $(12,36)$. Therefore, we measured the ability of WT or Y16F NS3-NS4A to block the nuclear translocation of endogenous IRF3 in response to SV. IRF3 translocated to the nucleus in $8 \%$ of the SV-infected cells, as measured by immunofluorescence assay (Fig. 5A and B). While the NS3-NS4A WT blocked nearly all of this nuclear translocation, NS3-NS4A Y16F only partially blocked this nuclear translocation, compared to the SV-infected cells in the absence of HCV protein (Fig. 5A and B), revealing that NS3-NS4A Y16F has a reduced ability to inhibit IRF3 activation.

To test whether NS3-NS4A Y16F similarly did not block IRF3 activation in the context of HCV replication, we utilized the HCV replicon system, which activates RIG-I signaling but prevents the transduction of IRF3 signaling by NS3-NS4A cleavage of MAVS, to prevent HCV- or SV-induced innate immune signaling (36). We infected control cells and cells stably expressing either WT or Y16F subgenomic replicons with SV and then measured induction of IFN- $\beta$ and several interferon-stimulated genes (ISGs) by RTqPCR. While the WT HCV replicon prevented SV-mediated induction of all ISGs tested, the HCV Y16F replicon did not block the induction of IFNB1, IFNL1, IFITM1, and RSAD2, and only partially blocked the induction of several other ISGs (Fig. 5C). Taken together, these data reveal that the Y16F substitution prevents NS3-NS4A from fully blocking IRF3 activation and signaling in response to viral infection.

TBK1 inhibition restores HCV Y16F viral replication to the level of HCV WT. Since IRF3 deletion was able to restore Y16F viral replication to the level of HCV WT, we wanted to determine whether the signaling pathway regulated by $Y 16$ would require TBK1 or IKK $\varepsilon$, the kinases that phosphorylate and activate IRF3. To this end, we utilized 

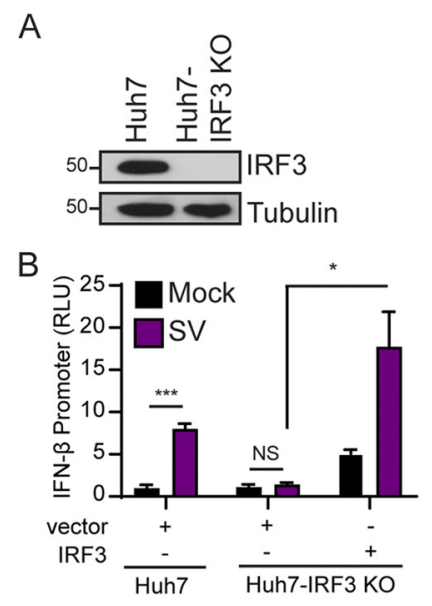
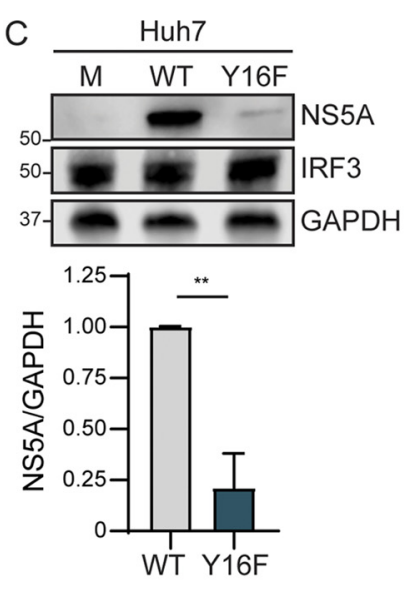

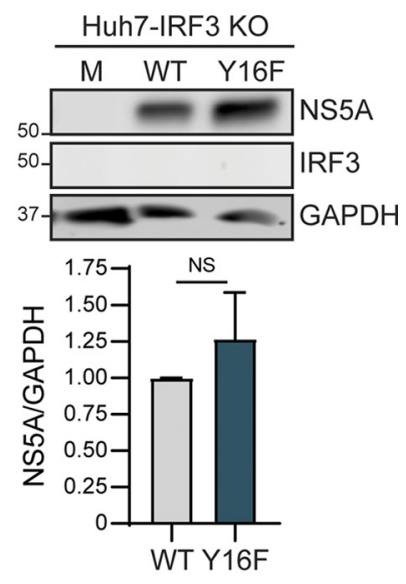

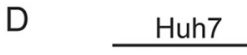

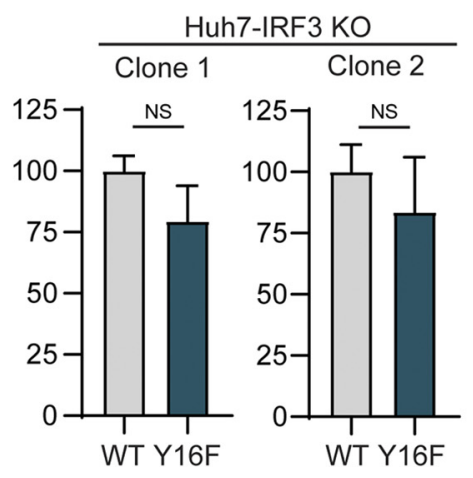

FIG 4 IRF3 deletion in Huh7 cells restores HCV Y16F replication to the level of HCV WT. (A) Immunoblot of extracts of Huh7 and Huh7-IRF3 KO cells. (B) IFN- $\beta$ promoter reporter luciferase expression of Huh7 and Huh7-IRF3 KO cells expressing either vector or IRF3 that were either mock or SV infected $(20 \mathrm{~h})$. Values show means \pm the SD ( $n=$ three technical replicates) in RLU. (C) Immunoblot analysis of lysates harvested at 72 hpi from Huh7 and Huh7-IRF3 KO cells infected with HCV, WT, or NS4A Y16F (JFH1, MOI of 0.3). Graphs below each blot show quantification of NS5A protein relative to GAPDH (means \pm the SEM; $n=$ three biological replicates). (D) Focus-forming assay of supernatants harvested at $72 \mathrm{hpi}$ from Huh7 or Huh7-IRF3 KO cells (clones 1 and 2) infected with HCV, WT, or NS4A Y16F (MOI of 0.3). The data are presented as the percent HCV titer from the Y16F strain relative to the WT (set at $100 \%)$ and show means \pm the SEM ( $n=$ two biological replicates). Data were analyzed by Student $t$ test $\left({ }^{*}, P<0.05 ;{ }^{* *}, P<0.01 ;{ }^{* * *}, P<0.005 ; \mathrm{NS}\right.$, not significant).

the TBK1/IKK $\varepsilon$ inhibitor, BX795 (37). First, we verified that the treatment of BX795 blocks phosphorylation of TBK1 ( $p$-TBK1) induced by SV by immunoblotting (Fig. 6A). We then treated Huh7 cells with either dimethyl sulfoxide (DMSO) or BX795, mock infected or infected with HCV WT or Y16F, and then measured NS5A protein expression from lysates harvested at $72 \mathrm{~h}$ postinfection. Although the level of NS5A expression of the Y16F virus in the DMSO-treated cells was reduced compared to the WT virus, as seen before in the parental Huh7 cells, the levels were similar in the BX795-treated cells (Fig. $6 \mathrm{~B})$, indicating that $\mathrm{Y} 16$ regulates a TBK1-dependent signaling pathway that can inhibit HCV infection.

Riplet interaction with HCV NS4A is reduced by the Y16F mutation. Our data described thus far reveal that NS4A Y16 regulates NS3-NS4A inhibition of TBK1/IKK $\varepsilon^{-}$ IRF3-mediated antiviral signaling. This IRF3-mediated signaling, which limits HCV replication, is RIG-I signaling independent and MAVS cleavage independent. Together, these data suggest: (i) that there is a factor that induces signaling to IRF3 that is targeted by NS4A Y16 (and not Y16F) and (ii) that this factor is present in Huh7 cells but absent or nonfunctional in Huh-7.5 cells. NS3-NS4A cleaves and inactivates three known host proteins involved in the IRF3 signaling axis: MAVS, TRIF (the TLR3 signaling adaptor), and Riplet $(10,12,19,24,25,36)$. Since we have demonstrated that NS3-NS4A Y16F cleaves MAVS (Fig. 3), and it is known that Huh7 cells do not have functional TLR3 
A
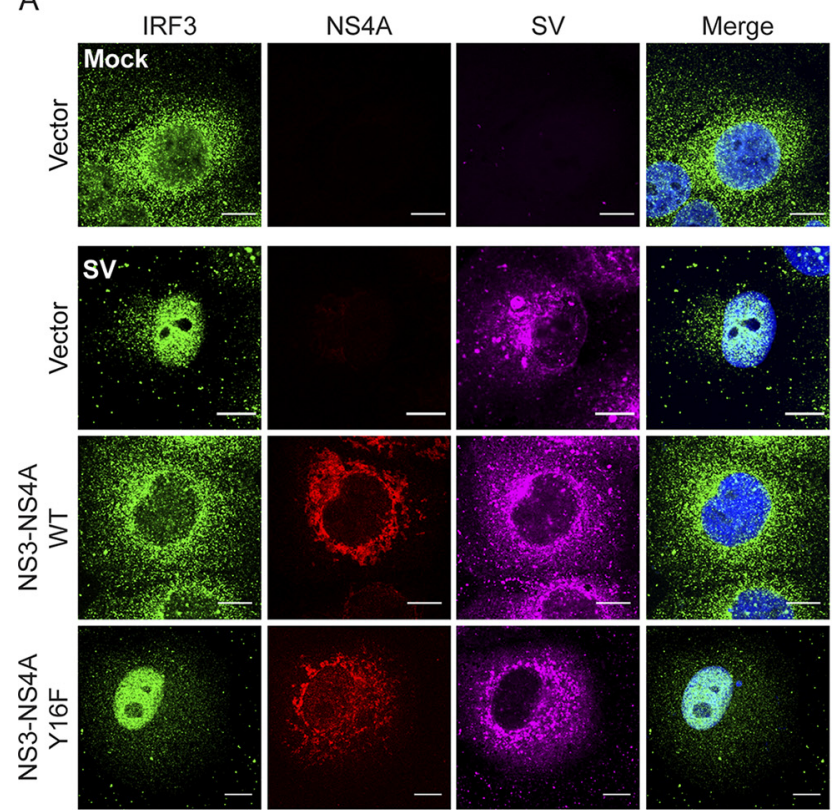

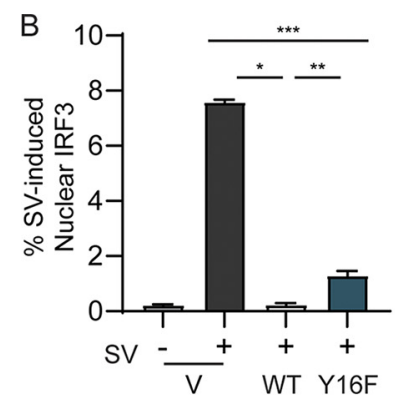

C

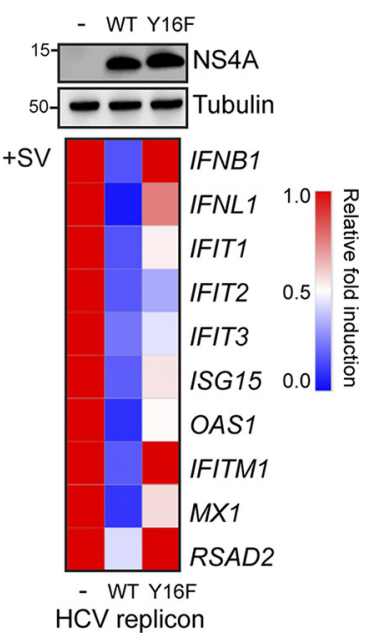

FIG 5 HCV NS3-NS4A Y16F does not block IRF3 activation. (A) Confocal micrographs of Huh7 cells expressing either NS3-NS4A WT or Y16F (genotype 1B), or vector, that were either mock or SV infected (20 h) and immunostained with anti-IRF3 (green), anti-NS4A (red), or anti-SV (magenta). Nuclei were stained with Hoechst (blue). Scale bar, 10 $\mu \mathrm{m}$. (B) Quantification of the percent cells positive for SV (and either WT or Y16F NS3-NS4A, as indicated) that had nuclear IRF3. The data are displayed as means \pm the SEM $(n=$ two biological replicates of $>500$ cells counted under each condition and replicate). Data were analyzed by one-way ANOVA $\left(^{* *}, P<0.01{ }^{* * *}, P<0.005\right)$. (C) Immunoblot analysis of lysates from Huh7(-), Huh7-HP WT replicon, or Huh7-HP Y16F replicon cells, and a heat map (below) that shows the mean relative fold induction (SV infected/mock infected, relative to HPRT1) of specific genes, as measured by RT-qPCR analysis of RNA from mock- or SV-infected $(20 \mathrm{~h})$ Huh7, Huh7-HP WT replicon, or Huh7-HP Y16F replicon cells from three biological replicates.

signaling (38), we hypothesized that the E3 ubiquitin ligase Riplet may be differentially regulated by NS3-NS4A WT and Y16F. Interestingly, we found that Huh-7.5 cells express reduced levels of Riplet protein and Riplet (RNF135) mRNA compared to Huh7 cells (Fig. $7 \mathrm{~A}$ and $\mathrm{B})$. This low level of Riplet likely reduces its ability to drive functional signaling responses during infection. Therefore, we tested whether Riplet ectopic expression in Huh-7.5 cells could limit HCV Y16F replication relative to HCV WT. We generated Huh-7.5 cells expressing V5-tagged Riplet (Fig. 7B and C), infected these cells with HCV WT or Y16F, and measured HCV NS5A expression. In Huh-7.5+Riplet-V5 cells, but not Huh-7.5 cells, HCV Y16F replication was reduced compared to WT (Fig. 7C). Similarly, the amount of infectious virus generated in the Huh-7.5+Riplet-V5 cells or Huh7 cells from the HCV Y16F virus was also much lower than WT ( 90\% lower in each), but in the Huh-7.5 cells the level of Y16F virus was still only partially reduced compared to WT, similar to before ( $\sim 50 \%$ lower) (Fig. $7 \mathrm{C}$, Fig. 2). Further, to determine whether the reduction of $\mathrm{Y} 16 \mathrm{~F}$ virus replication was a result of an inability of $\mathrm{Y} 16 \mathrm{~F}$ to block induction of the IFN response, we analyzed mRNA expression of a subset of ISGs and found that in Y16F-infected cells, which have lower levels of virus, the expression of these ISGs is somewhat increased relative to WT-infected cells (Fig. 7D). We note that the overall levels of HCV replication (both WT and Y16F) in the Huh-7.5+ Riplet-V5 cells were lower than those seen in the parental Huh-7.5 cells, likely due to the higher levels of Riplet expression in these cells (Fig. 7A and B) and the known role of Riplet in inhibiting HCV replication (19). 

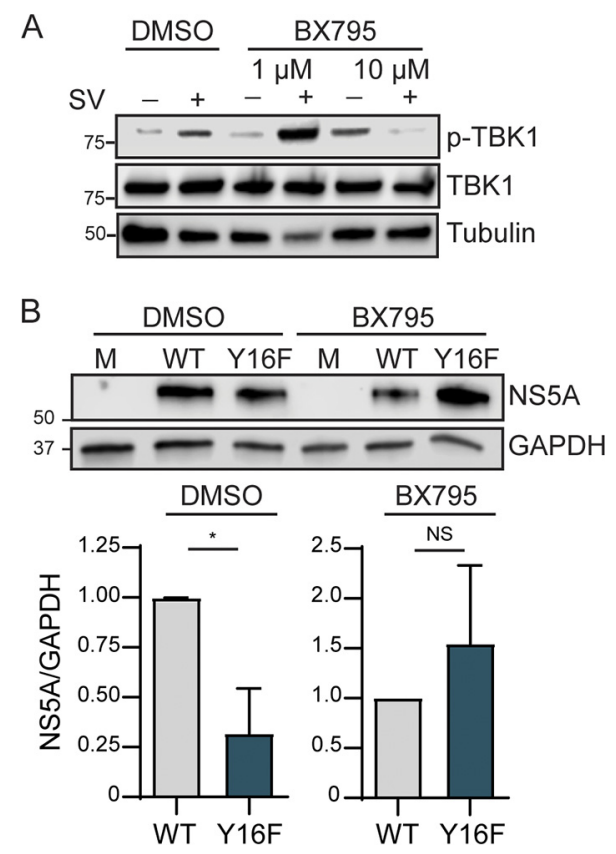

FIG 6 TBK1 inhibition restores HCV Y16F viral replication to the level of HCV WT. (A) Immunoblot analysis of lysates harvested from DMSO-treated or BX795 (1 or $10 \mu \mathrm{M})$-treated Huh7 cells that were either mock-infected (M) or infected with SV (20 h). (B) Immunoblot analysis of lysates harvested at $72 \mathrm{hpi}$ from Huh7 cells either pretreated for $1 \mathrm{~h}$ with DMSO or with BX795 (10 $\mu \mathrm{M})$, infected with HCV, WT, or NS4A Y16F (JFH1, MOI of 0.3). Graphs below each blot show quantification of NS5A protein relative to GAPDH (means \pm the SEM; $n=$ three biological replicates). Data were analyzed by Student $t$ test ${ }^{*}, P<0.05 ; \mathrm{NS}$, not significant).

To test the role of NS4A Y16 in targeting Riplet, we first examined the localization of overexpressed NS3-NS4A WT or Y16F with HA-tagged Riplet in Huh7 cells by immunofluorescence. Similar to others, we did not detect any major difference in the localization of NS4A WT or Y16F (5). In cells expressing NS3-NS4A WT, we found that Riplet was localized in small, punctate aggregates throughout the cytoplasm, whereas in cells expressing NS3-NS4A Y16F, Riplet was diffusely localized throughout the cytoplasm, similar to that seen in vector-expressing cells and described previously (18) (Fig. 8A). We also found that in cells expressing NS3-NS4A WT, but not Y16F, Riplet and NS4A were in close proximity to each other (Fig. 8A, zoom), suggesting that NS4A may interact with Riplet in a Y16-dependent manner. Indeed, we found that NS4A alone interacted with Flag-tagged Riplet and that the $\mathrm{Y} 16 \mathrm{~F}$ mutation reduced this interaction by approximately 70\% (Fig. 8B). Taken together, these data suggest that the NS4A Y16 residue is necessary for the ability of NS3-NS4A to interact with Riplet and to block antiviral innate immune signaling during HCV infection.

The RING domain of Riplet regulates HCV Y16F virus replication. Riplet consists of three domains: an N-terminal RING domain, a predicted central coil-coil region, and a C-terminal PRY-SPRY motif $(18,20)$. In general, RING domains of E3 ubiquitin ligases are required for interactions with E2 ubiquitin conjugating enzymes. As such, loss of the RING domain in an E3 ubiquitin ligase would prevent the ability of this ligase to conjugate K63-linked ubiquitin to its substrates. Thus, to determine whether the RING of Riplet is required to limit HCV replication, we generated pools of Huh-7.5 cells overexpressing a Riplet lacking the RING domain (Flag-Riplet $\triangle R I N G$ ) or full-length Riplet (Flag-Riplet FL) (Fig. 9A). We then either mock infected or infected cells with HCV WT or Y16F viruses, and then measured NS5A protein expression in lysates harvested at $72 \mathrm{~h}$ postinfection. We found that, as previously observed (Fig. 7), Y16F viral replication is reduced nearly $50 \%$ compared to HCV WT in full-length Riplet-expressing cells. However, in the Riplet $\triangle \mathrm{RING}$-expressing cells, Y16F viral replication phenocopies that seen in Huh-7.5 cells, with nearly similar replication of both HCV WT and Y16F 


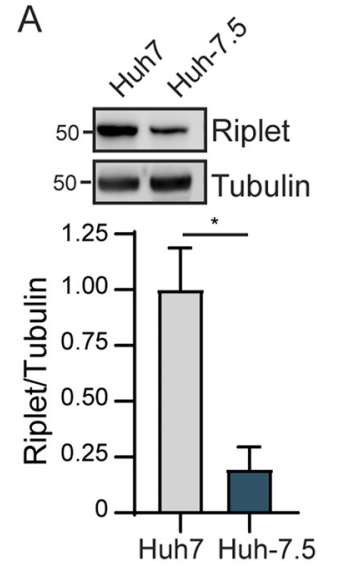

B

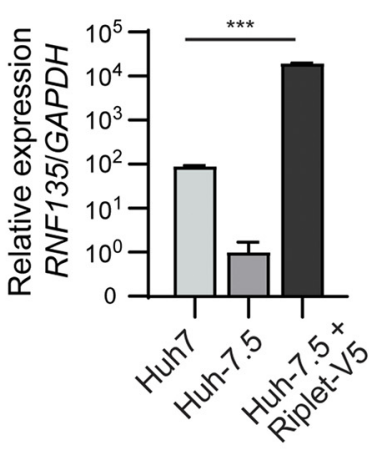

C$$
\text { Huh7 }
$$
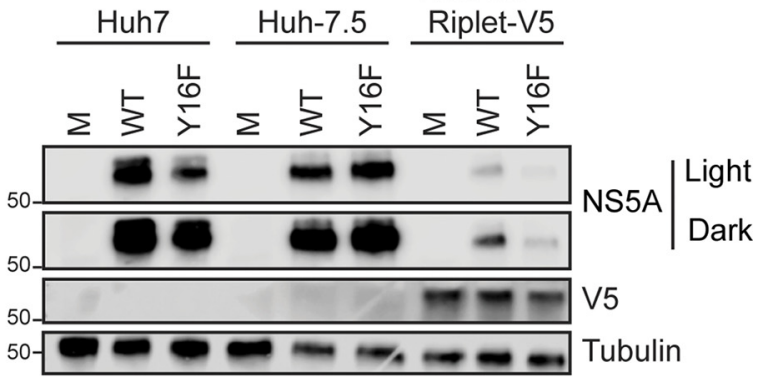

$$
\text { so }
$$
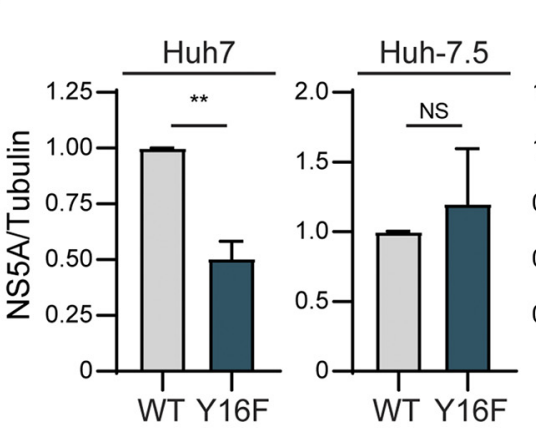

Huh-7.5+

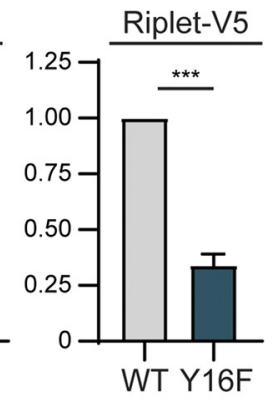

D

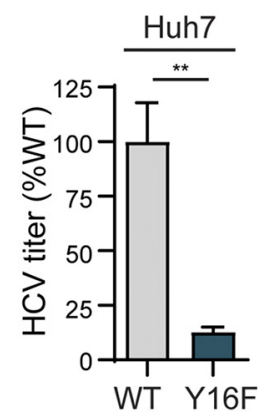

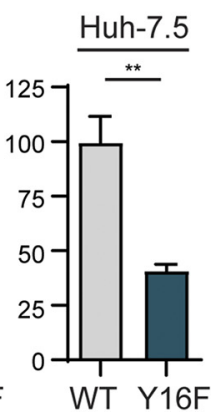

Huh-7.5 +

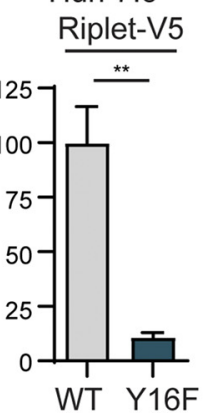

E

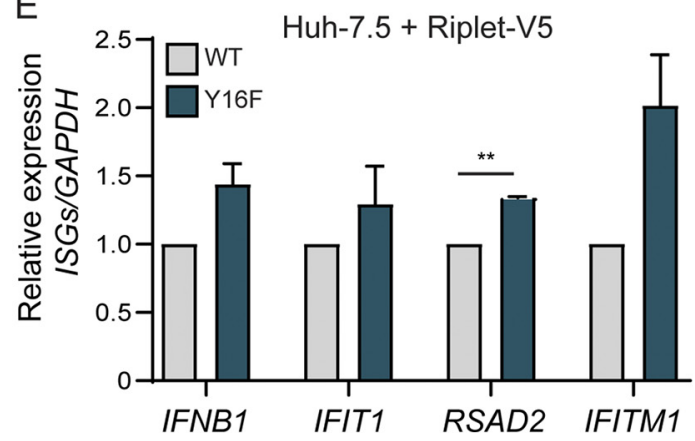

FIG 7 Overexpression of Riplet reduces HCV NS4A Y16F replication in Huh-7.5 cells. (A) Immunoblot analysis of lysates harvested from Huh7 and Huh-7.5 cells for endogenous Riplet protein expression. The graph below the blot shows the means \pm the SEM ( $n=$ three biological replicates) of quantification of Riplet protein expression relative to tubulin. (B) RNF135 (Riplet) expression relative to GAPDH from Huh7, Huh-7.5, and Huh-7.5 + Riplet-V5 cells, as analyzed by RT-qPCR, with data displayed as means \pm the SD ( $n=$ two to three technical replicates). Data were analyzed by one-way ANOVA analysis across the means of the three groups (***,P<0.005). (C) Immunoblot analysis of lysates harvested from the indicated cell lines infected with HCV, WT, or NS4A Y16F (JFH1, MOI of 0.3) or mock infected (M) at 72 hpi. Two different exposures (light and dark) are shown for NS5A. Graphs below each blot show means \pm the SEM ( $n=$ three biological replicates) of quantification of NS5A protein relative to tubulin. (D) Focus-forming assay of supernatants harvested at 72 hpi from the indicated cell lines infected with HCV, WT, or NS4A Y16F (MOI of 0.3). The data are presented as the percent HCV titer from the Y16F strain relative to the WT (set at $100 \%$ ) and show means \pm the SEM ( $n=$ three biological replicates). (E) RT-qPCR analysis of the indicated IFN-stimulated genes from RNA harvested from Huh-7.5+Riplet-V5 cells infected with HCV, WT, or NS4A Y16F (JFH1, MOI of 1) at 48 hpi. The data are normalized to Huh-7.5+Riplet-V5 mock-infected cells, are presented as the induction of indicated genes (relative to HPRT1 with the WT level set at 1$)$, and display means \pm the SEM ( $n=$ two biological replicates). Data were analyzed by Student $t$ test $\left({ }^{*}, P<0.05\right.$; ${ }^{* *}, P<0.05 ;{ }^{* * *}, P<0.005 ; \mathrm{NS}$, not significant).

(Fig. 9B). Taken together, these data suggest that the RING domain of Riplet is important for its ability to restrict Y16 viral replication and block downstream antiviral signaling during HCV infection.

\section{DISCUSSION}

Our results identify a new antiviral signaling program regulated by HCV NS3-NS4A. We found that mutation of NS4A Tyr-16 to phenylalanine, in both the context of an HCV subgenomic RNA replicon and in the context of fully infectious HCV, results in reduced viral replication in Huh7 cells, but not in Huh-7.5 cells. We show that both NS3-NS4A WT and Y16F cleave MAVS. Further, we found that Huh-7.5 cells, in addition to lacking RIG-I signaling (15), express low levels of Riplet (Fig. 6). Importantly, ectopic expression of 

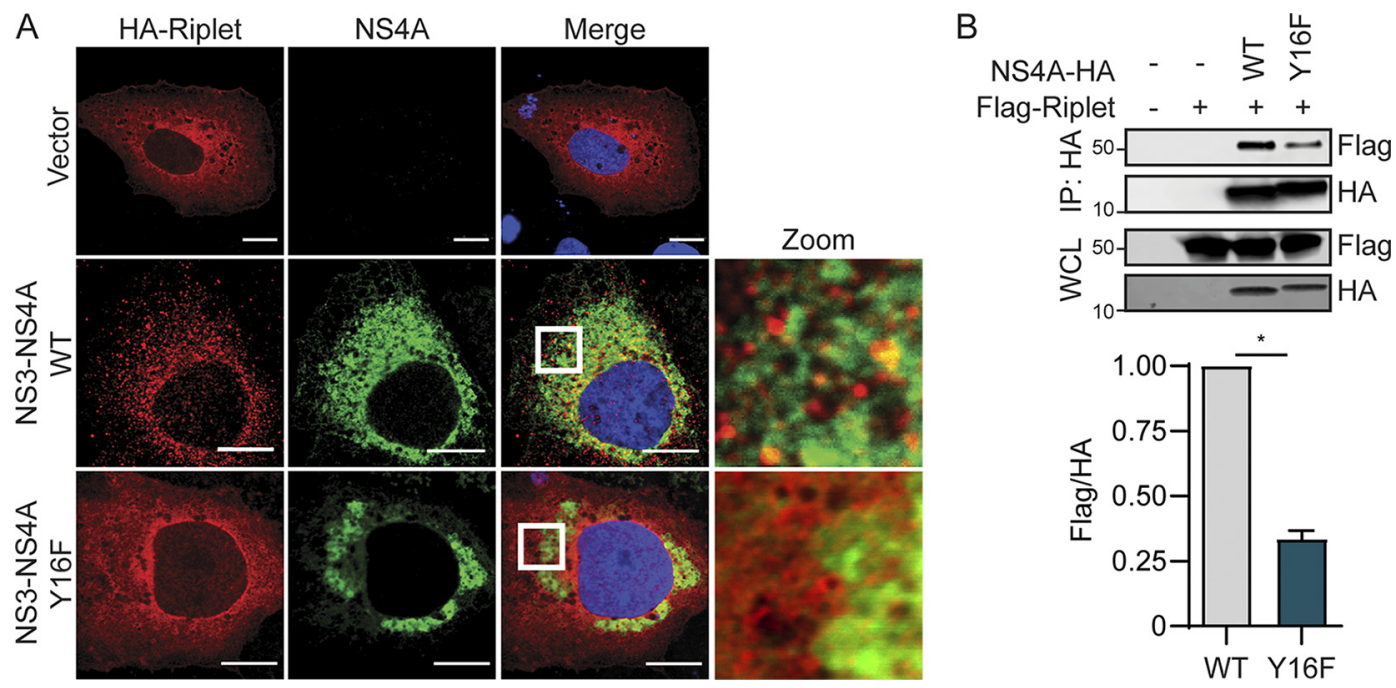

FIG 8 Riplet interaction with HCV NS4A is reduced by the Y16F mutation. (A) Confocal micrographs of Huh7 cells expressing HA-Riplet and either NS3-NS4A WT or Y16F (genotype 1B), or vector, that were immunostained with anti-NS4A (green) and anti-HA (red), with the nuclei stained with Hoechst (blue). The zoom panel is taken from the images in the white boxes. Images are representative of $\sim 50$ cells analyzed. Scale bar, $10 \mu \mathrm{m}$. (B) Immunoblot analysis of anti-HA (NS4A) immunoprecipitated extracts or whole-cell lysate (WCL) from Huh7 cells transfected with plasmids expressing Flag-Riplet and NS4A-HA (genotype 1B) WT or Y16F, or vector (-). The graph directly below shows means \pm the SEM ( $n=$ three biological replicates) of the relative fold change of Flag-Riplet to NS4A-HA in the immunoprecipitated lanes. Data were analyzed by Student $t$ test $\left({ }^{*}, P<0.05\right)$.

Riplet in Huh-7.5 cells resulted in reduced replication of HCV Y16F compared to WT virus. We also found that NS4A WT binds to Riplet, while NS4A Y16F does not bind as well. Taken together, this supports the model that HCV inactivates Riplet to prevent signaling to IRF3 and an antiviral response that can inhibit HCV replication. Our work reveals that the NS3-NS4A Y16 residue plays a critical role in the inactivation of this signaling pathway. Thus, NS4A Y16 regulates an antiviral signaling program activated by a Riplet-IRF3-dependent, but RIG-I-MAVS-independent, signaling axis.

We found that HCV containing an NS4A Y16F substitution in two HCV genotypes, either the JFH1 genotype 2A virus or the HP genotype 1B subgenomic replicon, has lower levels of replication than WT in Huh7 cells (Fig. 1B, Fig. 2A and B), but that both

A

Huh-7.5+ Flag-Riplet

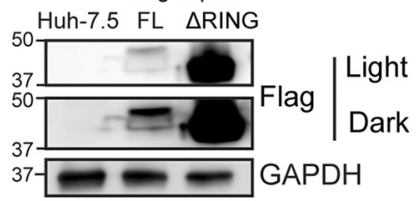

B

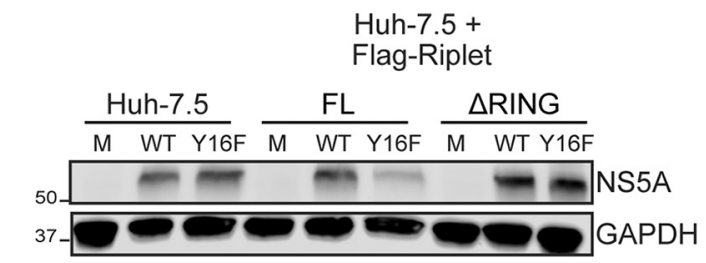

Huh-7.5+

Huh-7.5+

Huh-7.5
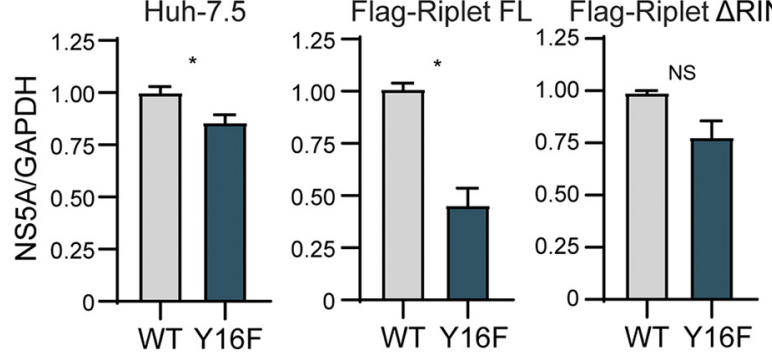

FIG 9 The RING domain of Riplet regulates HCV Y16F virus replication. (A) Immunoblot analysis of Huh-7.5 cells expressing Flag-Riplet full-length (FL) or Flag-Riplet $\triangle$ RING. Two different exposures (light and dark) are shown for Flag. (B) Immunoblot analysis of lysates harvested at 72 hpi from Huh-7.5, Huh-7.5+Flag-Riplet FL, and Huh7.5+Flag-Riplet $\triangle$ RING cells infected with HCV, WT, or NS4A Y16F (JFH1, MOI of 0.3). Graphs below the blot show quantification of NS5A protein relative to GAPDH (means \pm the SEM; $n=$ three biological replicates). 
the WT and Y16F viruses have similar levels of replication in Huh-7.5 cells or in Huh7-IRF3 KO cells (Fig. 2B, Fig. 4C and D). Although we did find that in experiments that assessed viral titer, the $\mathrm{Y} 16 \mathrm{~F}$ virus from Huh-7.5 cells had a reduced viral titer compared to the WT, this reduction $(\sim 50 \%)$ was not as much as that of virus harvested from the Huh7 parental, Huh7-RIG-I KO, or Huh-7.5+Riplet-V5 cells ( $~ 80 \%)$. This, along with our replication experiments, suggests that while the $\mathrm{Y} 16 \mathrm{~F}$ substitution does not itself directly affect the functions of the HCV protease in replication, including HCV polyprotein processing, NS3 helicase function, or viral assembly and envelopment, the virus with this substitution may still be inhibited by the low, remaining levels of Riplet present in the Huh-7.5 cells that we use to measure the production of infectious virus. Indeed, the viral titers between the WT and Y16F viruses harvested from the Huh7-IRF3 $\mathrm{KO}$ cells were similar to each other (Fig. 4D). Similar to our findings, Kohlway et al. found that the replication of a genotype $2 \mathrm{~A}$ subgenomic replicon (pYSGR-JFH1/GLuc) containing this $\mathrm{Y} 16 \mathrm{~F}$ substitution was not altered in Huh-7.5 cells (7), whereas Brass et al. did find reduced replication of a Y16F genotype 1B subgenomic replicon [pCon1/SG$\mathrm{Neo}(\mathrm{I}) / \mathrm{AflII}$ in Huh-7.5 cells (5). While it is unclear what mediates the difference in our $\mathrm{HCV}$ replication results from those of Brass et al., it is possible that this could be due to differences in the replication fitness of the replicons used, that Huh-7.5 cells from different labs do not have the same expression levels of Riplet, or that there are other genetic differences between the cell lines used. Unfortunately, our attempts to use CRISPR to delete Riplet from Huh7 cells resulted in altered cellular growth. Nevertheless, since we found that the Y16F substitution does not affect either the interaction of NS4A with NS3, processing of the NS3-NS4A junction, or MAVS cleavage, our results suggest that it has a specific role in targeting NS3-NS4A to Riplet.

The mechanisms by which the HCV protease targets and inactivates Riplet are not entirely clear. Riplet is an E3 ubiquitin ligase localized in the cytoplasm that activates RIG-I by both binding and adding K63-linked ubiquitin chains to it $(20,39)$. Although others have concluded that NS3-NS4A cleaves Riplet in the first amino acid of its RING domain resulting in its destabilization (19), we were not able to detect a Riplet cleavage product or a reduction in Riplet protein abundance by immunoblot analysis upon overexpression of NS3-NS4A in cells, although we cannot rule out this possibility. While it is possible that NS3-NS4A inactivation of Riplet via cleavage may result in its destabilization, analogous to how NS3-NS4A cleavage of TRIF accelerates its proteolysis (25), it is also possible that simply the binding of NS3-NS4A to Riplet can inactivate it. Indeed, we did find that the localization of Riplet changed from cytoplasmic to punctate, often near NS4A, following overexpression of NS3-NS4A WT, but not Y16F, which could either represent a differential localization as a result of binding to NS4A to prevent Riplet function or represent cleavage by the WT NS3-NS4A (Fig. 8A). Indeed, the dengue virus protease cofactor NS2 (analogous to HCV NS4A) inactivates cGAS simply by binding to it and inducing its autophagic degradation (40). In addition, the influenza A virus NS1 protein inactivates Riplet by binding to it (41). While we have shown that NS4A Y16F interaction with Riplet is reduced compared to NS4A WT, NS3-NS4A inactivation of Riplet may also be a result of indirect mechanisms. Therefore, while it is clear that NS3-NS4A inactivates Riplet, further studies are needed to determine the exact mechanisms by which this occurs.

While HCV NS4A anchors the NS3-NS4A protease to intracellular membranes (6), we do not yet know the mechanisms by which the Y16F substitution in NS4A would specifically alter Riplet localization to alleviate the block to Riplet signaling. Similar to others, we did not find that the Y16F substitution altered the localization of NS4A within membranes (5). Since NS4A can bind Riplet in the absence of NS3, it is possible that NS4A Y16 is simply required for Riplet binding, either directly or through other proteins. In fact, as the hydroxyl group of this tyrosine residue in NS4A is positioned such that it interacts with the phospholipid head groups of the membrane bilayer, while a phenylalanine at the position would be missing this hydroxyl group, Y16 may be poised to mediate protein-protein interactions directly with Riplet or with accessory binding proteins (5). We also note that it is possible that phosphorylation of NS4A Y16 
could regulate these protein-protein interactions. Thus, NS4A Y16 likely mediates interactions with Riplet to prevent Riplet from interacting with proteins that promote antiviral innate immune signaling.

Our results suggest that HCV activates a Riplet-dependent signaling cascade to IRF3 that is independent of both RIG-I and MAVS. The following pieces of evidence presented here support the existence of this pathway: (i) NS3-NS4A WT and Y16F both cleave MAVS (Fig. 3); (ii) Y16F cannot bind to Riplet as well as WT (Fig. 8); (iii) NS3-NS4A WT, but not Y16F, blocks SV-mediated IRF3 activation and induction of ISGs (Fig. 2 and 5); (iv) WT and Y16F viruses only grow equivalently to each other in cells that lack both RIG-I and Riplet or lack IRF3 (Fig. 1, 2, and 4); and (v) overexpression of Riplet in cells without RIG-I signaling can reduce Y16F viral replication (Fig. 7 and 9). While the identification of this RIG-I-MAVS independent signaling cascade that induces IRF3 activation and IFN- $\beta$ was surprising to us, others have shown that infection of RIG-I KO mouse embryonic fibroblasts with vesicular stomatitis virus, known to be sensed by only RIG-I (42), does result in a small induction of IFN- $\beta$ mRNA, even though other stimuli do not induce IFN- $\beta$ in these cells (20). Thus, it is possible that in our human Huh7-RIG-I KO cells, ISGs are induced during HCV infection to limit Y16F viral replication. Further, regulation of HCV replication may require a combination of factors, such as downstream signaling driven by T55 in the RIG-I CARD and Riplet, as well as other yet-to-be-discovered factors. Overall induction of this Riplet-IRF3 signaling pathway in the absence of RIG-I signaling is likely stimulus dependent and cell type dependent.

We do not yet know the full identity of this Riplet-IRF3 signaling cascade regulated by NS3-NS4A Y16. We predict that Riplet is either directly adding K63-linked ubiquitin chains to signaling proteins in this pathway or that it interacts with these signaling proteins to activate them, as it does with RIG-I $(18,20)$. The only known Ripletinteracting protein that is $\mathrm{K} 63$ ubiquitinated is RIG-I. The Riplet-signaling target is likely not MDA5 because it is not a Riplet substrate $(18,20)$, and the Y16F protease cleaves MAVS, the MDA5 downstream signaling adaptor. It could be the IRF3 kinases TBK1 and IKK $\varepsilon$ or some other unknown upstream factor $(43,44)$. Future studies are needed to further identify the Riplet-interacting proteins that activate this noncanonical antiviral signaling pathway.

Here, we identify a new, noncanonical branch of an antiviral signaling pathway regulated by NS3-NS4A that can inhibit HCV infection. This signaling pathway is driven by Riplet, through its RING domain, to induce TBK1/IKK $\varepsilon$-mediated IRF3 activation, and our data suggest that it does not require MAVS. This signaling results in the transcriptional induction of IRF3-regulated genes, including IFNB1 and several ISGs. Ultimately, full characterization of this novel pathway may reveal insights into antiviral innate immunity to other RNA viruses, such as influenza A virus, which inactivates Riplet (41). In summary, our work identified a specific amino acid in NS4A of which mutation can uncouple Riplet inactivation from MAVS cleavage and HCV replication. By studying HCV replication in the context of this specific mutation in NS4A, we learned that Riplet can be regulated independently from RIG-I and MAVS during HCV infection and that, in addition to its known innate immune regulatory functions, NS3-NS4A also regulates a Riplet/IRF3-dependent, RIG-I-MAVS-independent branch of an antiviral signaling pathway that limits HCV infection.

\section{MATERIALS AND METHODS}

Cell culture. Huh7 and Huh-7.5 (15) cells (a gift from Michael Gale, Jr., University of Washington), as well as 293T cells (ATCC; CRL-3216), were grown at $37^{\circ} \mathrm{C}$ with $5 \% \mathrm{CO}_{2}$ in Dulbecco modified Eagle medium (DMEM; Mediatech) supplemented with 10\% fetal bovine serum (FBS; HyClone), and $25 \mathrm{mM}$ HEPES (Thermo Fisher), referred to as complete DMEM (cDMEM). Huh7 and Huh-7.5 cells were verified using a Promega GenePrint STR kit (DNA Analysis Facility, Duke University), and all cells were tested and found to be Mycoplasma-free using a LookOut PCR detection kit (Sigma).

Plasmids and transfections. These plasmids were used in this study: pEF-NS3, pEF-NS3-NS4A (genotype 1B), and pEF-NS3-NS4A S1165A (45); pHCV-HP WT (containing the following 7 amino acid changes: NS3 [P1115L, K1609E], NS4B [Q1737R], NS5A [P2007A, L2198S, S2236P], and NS5B [V2971A]) and pHCV-HP $\triangle N S 5 B$ (46); pEF1-NS4A HA (9); pEF-Tak-Flag-MAVS (12); pCR-Bluntll-TOPO (Addgene, catalog no. 41824) (47); phCas9 (Addgene, catalog no. 41815) (47); pCMV-Renilla and pGL4.74[hRluc/TK] (Pro- 
mega); pIFN- $\beta$-Luc (48); pEF-Tak-Flag and pEF-Tak-Flag-RIG-I (49); pEGFP-C1-IRF3 (50); psJFHI-M9 WT (32); pX330 (Addgene, catalog no. 42230) (51); pcDNA-BLAST (52); pPAX2 and pMD2.G (Addgene catalog no. 35002 [53] and Addgene catalog no. 12259); pCCSB-Riplet-V5 (Dharmacon: NM_032322.4, cDNA clone MGC161700); pCAGGS-HA-Riplet (41) (Michaela Gack at the University of Chicago); and pCMV-Flag-IRF3 WT (54). psJFHI-M9 Y16F, pEF-NS3-NS4A Y16F, pEF1-NS4A-HA Y16F, and pHCV-HP Y16F were generated by using QuikChange site-directed mutagenesis (Stratagene) of psJFHI-M9, pEF-NS3-NS4A, and pHCV-HP, respectively. pEF-Tak-Flag-Riplet was generated by InFusion (Clontech) cloning of pCAGGS-HA-Riplet into pEF-Tak. To generate pEF-Tak-Flag-Riplet $\triangle R I N G$, a minigene containing the RING deletion (amino acids 21 to 67) was digested with Notl, treated with calf intestinal alkaline phosphatase (New England Biolabs), and ligated into Notl-digested pEF-Tak-Flag-Riplet with T4 DNA ligase (New England Biolabs). To generate the RIG-I CRISPR guide RNA plasmids (pCR-BluntII-Topo-sgRIGI-I and pCR-BluntII-Topo-sgRIGI-2), sgRNA oligonucleotides were annealed and inserted into Afll-digested pCR-Bluntll-Topo by Gibson Assembly (New England Biolabs). To generate the IRF3 and MAVS CRISPR guide RNA plasmids, sgRNA oligonucleotides were annealed and inserted into Bbsl-digested pX330 (pX330-sgIRF3; pX330-sgMAVS). All oligonucleotide sequences are listed in Table 1. The sequences of all plasmids were verified by DNA sequencing and are available upon request. DNA transfections were performed using FuGENE6 (Promega) according to manufacturer's instructions.

Generation of KO lines. Huh7-RIG-I knockout (KO) cells were generated by CRISPR/Cas9, using two guides targeting the intron before exon 1 (sgRNA 1) and within exon 1 (sgRNA 2) that were designed with the CRISPR design tool (http://crispr.mit.edu). pCR-BluntIl-Topo-sgRIGI-I and pCR-Bluntll-ToposgRIGI-2, along with phCas9, which expresses Cas9 and neomycin (G148) resistance, were transfected into Huh7 cells. Huh7-IRF3 KO cells were generated by CRISPR/Cas9 using a single guide that targets exon 2 (55), and Huh7-MAVS KO cells were generated by CRISPR/Cas9 using a single guide that targets exon 5 (56). To generate these cells, Huh7 cells were transfected with the respective guide containing plasmid (pX330-sgIRF3 or pX330-sgMAVS), along with pcDNA-BLAST (which encodes blasticidin resistance). In both cases, cells were replated the day after transfection at limiting dilutions into $15 \mathrm{~cm}$ dishes and then incubated with CDMEM containing either $0.4 \mathrm{mg} / \mathrm{ml}$ Geneticin (G418; Life Technologies) for 5 days or $0.2 \mu \mathrm{g} / \mathrm{ml}$ blasticidin for 3 days. Individual cell clones were then selected and expanded. Isolated clones were screened for either RIG-I, MAVS, or IRF3 protein expression by immunoblotting. Genomic DNA was isolated from candidate RIG-I or IRF3 KO cell clones using the QuickExtract DNA extraction solution (Epicentre). Genomic DNA isolated from the RIG-I, MAVS, or IRF3 KO cell clones was then amplified by PCR using primers spanning exon 1 for RIG-I, exon 5 for MAVS, or exon 2 for IRF3 (see Table 1). The resulting amplicons were cloned into PCR4-TOPO TA (Invitrogen) and Sanger sequenced. For RIG-I, all five of the sequenced genomic DNA clones had the start codon and exon 1 removed (four clones, 252-bp deletion; one clone, 250-bp deletion). For MAVS, all six of the sequenced genomic DNA clones contained a 128-bp insertion at the beginning of exon 5 that causes a frameshift resulting in a premature stop codon within exon 5. For IRF3, all five of the clones sequenced had a 4-bp deletion at the beginning of exon 2 that causes a frameshift resulting in a premature stop codon within exon 2 .

Generation of shRNA cell lines. Huh7 cells were either mock infected or infected with Sigma Mission TRC MAVS shRNA lentiviral particles (clone TRC N0000148945) or nontargeting control (Mission nontargeting shRNA; Sigma) at a multiplicity of infection (MOI) of 2 in CDMEM containing $8 \mu \mathrm{g} / \mathrm{ml}$ hexadimethrine bromide (Sigma). The following day, medium was replenished with cDMEM. At $48 \mathrm{~h}$ after lentiviral infection, CDMEM containing $2 \mu \mathrm{g} / \mathrm{ml}$ puromycin was added to the cells until the mock-treated cells died (3 days). Puromycin-resistant cells were harvested as pools, and cells were verified by immunoblot for MAVS.

Generation of Huh-7.5+Riplet-V5 cells. To generate Riplet-V5-expressing lentivirus, 293T cells were transfected with pCCSB-Riplet-V5, psPAX2, and pMD2.G. Supernatant was harvested at $48 \mathrm{~h}$ posttransfection and filtered through a $0.45-\mu \mathrm{m}$-pore size filter. Huh-7.5 cells were then infected with the Riplet-V5 lentivirus ( $500 \mu \mathrm{l}$ per well of a six-well plate), and the next day the virus was removed and replaced with cDMEM with $0.2 \mu \mathrm{g} / \mathrm{ml}$ blasticidin until the mock-transduced cells died ( 3 to 4 days). Blasticidin-resistant cells were harvested as pools, and cells were verified as transduced by immunoblotting for Riplet-V 5 and RT-qPCR analysis for RNF135 (Riplet).

Generation of Huh-7.5+Flag- $\triangle$ RING Riplet and Flag-Riplet cells. To generate Huh-7.5 cells expressing Flag-Riplet or Flag-Riplet $\triangle$ RING, we first linearized pcDNA-BLAST and pEF-Tak-Flag-Riplet with Bglll or Agel, respectively. Huh-7.5 cells were then cotransfected with the linearized plasmids using FuGENE6, and at $24 \mathrm{~h}$ posttransfection the medium was replaced with CDMEM containing $0.2 \mu \mathrm{g} / \mathrm{ml}$ blasticidin. Once the mock-transduced cells died ( 5 days), the blasticidin-resistant cells were harvested as pools, and cells were verified by immunoblotting for Flag to analyze Riplet expression.

HCV replicons. RNA was in vitro transcribed (MEGAscript T7 transcription kit; Thermo Fisher) from Scal-digested HP-HCV replicon plasmid DNA, either WT, Y16F, or $\triangle N S 5 B$. The in vitro-transcribed RNA was treated with DNase (Thermo Fisher), purified by phenol-chloroform extraction, and integrity verified on a denaturing gel. For electroporation, $1 \mu \mathrm{g}$ of HCV replicon RNA was mixed with $4 \times 10^{6}$ Huh7 or Huh-7.5 cells in cold $1 \times$ phosphate-buffered saline (PBS) in a 4-mm cuvette and then electroporated at $960 \mu \mathrm{F}$ and $250 \mathrm{~V}$ with a GenePulser XCell system (Bio-Rad). Electroporated cells were plated into 10-cm plates at $2 \times 10^{5}, 2 \times 10^{4}$, and $2 \times 10^{3}$ cells per dish, along with $2 \times 10^{5}$ cells that had been electroporated with $\triangle N$ S5B RNA. At $4 \mathrm{~h}$ postelectroporation, the cells were washed three times with $1 \times$ PBS and then once with CDMEM. At $24 \mathrm{~h}$ postelectroporation, the medium was changed to CDMEM supplemented with $0.4 \mathrm{mg} / \mathrm{ml} \mathrm{G} 418$. After 3 weeks of G418 selection, the cells were fixed and stained with crystal violet in $20 \%$ methanol. Colonies from triplicate plates were counted to determine the relative transduction efficiency, expressed as the percentage of Y16F colonies that were stably transduced relative to WT. 


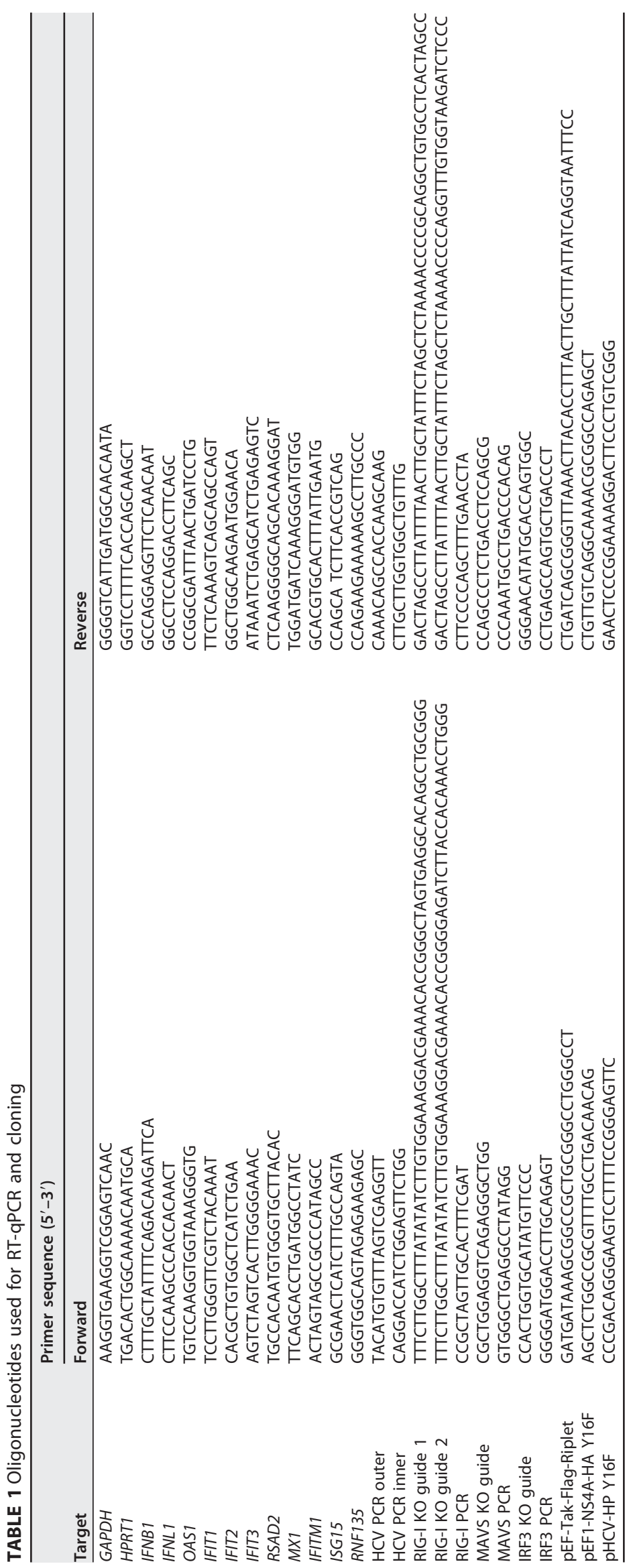


Huh7-HP WT and Huh7-HP Y16F replicon cell lines were generated by isolating and expanding single clones. The presence of the HCV replicon was determined by sequencing the NS4A-containing region following CDNA synthesis on extracted RNA (RNeasy RNA extraction kit; Qiagen) and PCR amplification of the NS4A region. The oligonucleotides used for PCR and sequencing are listed in Table 1.

HCV stock generation and infections. HCV JFH1-M9 WT and Y16F virus stocks were generated as described previously (32). The sequence of the virus at NS4A was confirmed after each passage by sequencing nested PCR products from generated CDNA using the oligonucleotides indicated in Table 1, as previously described (9). For HCV infections, cells were incubated in a low volume of serum-free DMEM containing virus at an $\mathrm{MOI}$ of 0.3 for 2 to $3 \mathrm{~h}$, after which the CDMEM was replenished. To quantify virus, cellular supernatants were analyzed by using a focus-forming assay.

Focus-forming assay. To measure the HCV titer, supernatants from infected cells were serially diluted and then used to infect naive Huh-7.5 cells in triplicate wells of a 48-well plate for $3 \mathrm{~h}$. At $48 \mathrm{~h}$ postinfection, the cells were washed with PBS and fixed with $4 \%$ methanol-free paraformaldehyde (Sigma) for $30 \mathrm{~min}$ and then washed again with PBS. The cells were then permeabilized $(0.2 \%$ Triton $\mathrm{X}-100$ [Sigma] in PBS), blocked (10\% FBS in PBS), and immunostained with a mouse anti-HCV NS5A antibody (1:500). Infected cells were visualized after incubation with horseradish peroxidase (HRP)conjugated secondary mouse antibody (1:500; Jackson ImmunoResearch) and a VIP peroxidase substrate kit (Vector Laboratories). Foci were counted at $\times 10$ magnification, and the viral titer was calculated using the following formula: (dilution factor $\times$ number of foci $\times 1,000$ )/volume of infection (in $\mu$ l), resulting in units of focus-forming units $/ \mathrm{ml}$.

Immunoblotting. Cells were lysed in a modified radioimmunoprecipitation assay (RIPA) buffer (10 mM Tris [pH 7.5], $150 \mathrm{mM} \mathrm{NaCl}, 0.5 \%$ sodium deoxycholate, 1\% Triton X-100) supplemented with protease inhibitor cocktail (Sigma) and phosphatase inhibitor cocktail (Millipore), and the postnuclear supernatants were harvested by centrifugation. The protein concentration was determined by a Bradford assay, and $10 \mu \mathrm{g}$ of quantified protein was resolved by SDS-PAGE, transferred to either polyvinylidene difluoride (for NS4A) or nitrocellulose membranes using either a Trans-Blot Turbo system (Bio-Rad) or a wet system (Bio-Rad), and blocked with either $3 \%$ bovine serum albumin (Sigma) in PBS with $0.1 \%$ Tween (PBS-T) or $10 \%$ FBS in PBS-T. Membranes were probed with specific antibodies against proteins of interest, washed three times with PBS-T, and incubated with species-specific HRP-conjugated antibodies (1:5,000; Jackson ImmunoResearch), washed again three times with PBS-T, and treated with Clarity enhanced chemiluminescence substrate (Bio-Rad). Membranes were then imaged on X-ray film or by using a LI-COR Odyssey FC. Immunoblots imaged using the LI-COR Odyssey FC were quantified with ImageStudio software, and raw values of the protein of interest were normalized to those of controls (either tubulin or GAPDH [glyceraldehyde-3-phosphate dehydrogenase], as indicated). For immunoblots developed on film, Fiji was used (57). ImageStudio and Fiji give similar quantification results when compared directly.

Immunoprecipitation. Quantified protein (between 80 and $160 \mu \mathrm{g}$ ) was incubated with proteinspecific antibodies (either R anti-HA [Sigma] or anti-NS4A) in PBS at $4^{\circ} \mathrm{C}$ overnight with head-over-tail rotation. The lysate-antibody mixture was then incubated with either protein $\mathrm{A}$ (for Flag-Riplet experiments) or protein G-Dynabeads (Invitrogen) for $2 \mathrm{~h}$. The beads were washed three times in either PBS or RIPA buffer for immunoprecipitation and then eluted in $2 \times$ Laemmli buffer (Bio-Rad) supplemented with $5 \% 2$-mercaptoethanol at $50^{\circ} \mathrm{C}$ for $5 \mathrm{~min}$. Proteins were resolved by SDS-PAGE and subjected to immunoblotting as described above.

Immunofluorescence analysis and confocal microscopy. Huh7 cells in four-well chamber slides were fixed in $4 \%$ formaldehyde, permeabilized with $0.2 \%$ Triton $X-100$, and immunostained with the following antibodies: mouse anti-HCV NS4A (genotype 1B, 1:100; Virogen), rabbit anti-HA (1:100; Sigma), rabbit anti-IRF3 (1:100; Cell Signaling Technology), and rabbit anti-SV (1:1,000; MBL International). Secondary antibody incubations were done with Alexa Fluor-conjugated antibodies (Thermo Fisher) and with Hoechst (Thermo Fisher) for $1 \mathrm{~h}$. After the antibody incubations, the slides were washed with $1 \times$ PBS and mounted with ProLong Gold Antifade mounting medium (Invitrogen). Samples were imaged on a Zeiss 780 upright confocal apparatus using a $63 \times / 1.25$ oil objective and the $405,488,561$, and 633 laser lines with pinholes set to $1 \mathrm{AU}$ for each channel (Light Microscopy Core Facility, Duke University). Imaging analysis was done using Fiji software (57).

Antibodies. Antibodies used for immunoblot and immunofluorescence analysis include: mouse anti-HCV NS4A (genotype 1B, 1:1,000; Virogen), mouse anti-HCV NS3 (genotype 1B, 1:1,000; Adipogen), mouse anti-HCV NS5A (genotype 2A, 1:1,000, clone 9e10; a gift from Charles Rice, Rockefeller University), mouse anti-Tubulin (1:5,000; Sigma), mouse anti-RIG-I (1:1,000; Adipogen), anti-Flag-HRP (1:2,500; Sigma), rabbit anti-Flag (1:1,000; Sigma), rabbit anti-MAVS (1:1,000; Bethyl Laboratories), mouse anti-IRF3 $(1: 1,000$; a gift from Michael Gale, Jr., University of Washington [35]), rabbit anti-IRF3 (1:100; Cell Signaling Technology), rabbit anti-TBK1 (1:1,000; Cell Signaling Technology), rabbit anti-phospho-TBK1 (1:1,000; Cell Signaling Technology), mouse anti-V5 (1:1,000; Sigma), mouse anti-HA (1:1,000; Sigma), rabbit anti-GAPDH (1:1,000; Cell Signaling Technology), Hoescht (1:500; Thermo Fisher), Alexa Fluor-conjugated secondary antibodies (1:500, Life Technologies), rabbit anti-SV (1:1,000, MBL International), and mouse anti-Riplet (1:500; a gift from Sun Hur, Harvard University [20]).

IFN- $\boldsymbol{\beta}$ promoter luciferase assays. IFN- $\beta$ promoter luciferase assays were performed by transfecting cells with pCMV-Renilla or pGL4.74[hRluc/TK], pIFN- $\beta$-Luc, and expression plasmids as indicated. The following day, the cells were infected with SV (Cantrell strain; Charles River labs). SV infections were performed in serum-free media at 200 hemagglutination units (HAU) for $1 \mathrm{~h}$, and then the complete medium was replenished. At $20 \mathrm{~h}$ postinfection, the cells were lysed, and a dual-luciferase assay was 
performed (Promega). Values are displayed as relative luciferase units (RLU), which normalizes the firefly luciferase (IFN- $\beta$-Luc) values to Renilla luciferase.

RT-qPCR. RNA was extracted from cell lysates using an RNeasy RNA extraction kit, and cDNA synthesis was performed on extracted RNA using iScript (Bio-Rad). The resulting CDNA was diluted (either 1:3 or 1:4) in $\mathrm{ddH}_{2} \mathrm{O}$. RT-qPCR analysis was performed using the Power SYBR green PCR master mix (Thermo Fisher) on the QuantStudio 6 Flex RT-PCR system. The oligonucleotide sequences used for RT-qPCR are listed in Table 1. Heat map analysis was generated using Morpheus software from the Broad Institute (https://software.broadinstitute.org/morpheus). First, the $2^{\Delta \Delta C T}$ values (comparative $C_{T}$ method) were calculated by setting the mock-infected Huh7 sample $C_{T}$ value as the baseline for each biological replicate. Then, the mean of the SV-infected Huh7 triplicate samples is set to 1 , and the relative fold induction for each gene between samples is shown. For HCV RT-qPCR, viral RNA was isolated from cells using an RNeasy kit (Qiagen). The HCV copy number was measured in triplicate by using a TaqMan one-step assay (TaqMan Fast Virus 1-Step Mix; Qiagen) with an HCV-specific probe targeting the 5' untranslated region of HCV (assay identifier Pa03454801_s1), as previously described (58). The copy number of HCV was calculated by comparison to a standard curve of a full-length in vitro-transcribed HCV RNA.

BX795 inhibitor treatment. Huh7 cells were pretreated with either DMSO or $10 \mu \mathrm{M}$ BX795 (Invivogen) for $1 \mathrm{~h}$ prior to HCV infection. After incubation with DMSO or BX795, medium was removed, and cells were infected at an MOI of 0.3 with either HCV WT or Y16F viruses in serum-free DMEM as described above. At $48 \mathrm{~h}$ postinfection, the cells were treated again with either DMSO or BX795 for $1 \mathrm{~h}$, and then the CDMEM was replenished. The following day ( $72 \mathrm{~h}$ postinfection), the lysates were harvested for immunoblotting.

Statistical analysis. A Student unpaired $t$ test or one-way analysis of variance (ANOVA) was implemented for statistical analysis of the data using GraphPad Prism software. Graphed values are presented as means \pm either the standard deviations (SD) or the standard errors of the mean (SEM [ $n=3$ or as indicated]; $\left.{ }^{*}, P \leq 0.05 ;{ }^{* *}, P \leq 0.01 ;{ }^{* * *}, P \leq 0.005\right)$.

\section{ACKNOWLEDGMENTS}

We thank the members of the Horner Lab and Heather Vincent for discussions and reviews of the manuscript, as well as Nicholas Barrows, Moonhee Park, Kevin Labagnara, Michelle Kim, Bianca Lupan, and Jason Willer for assistance with experiments, and the Duke University Light Microscopy Core Facility and the Duke Functional Genomics Core Facility. We also thank the following individuals for reagents: Charles Rice at Rockefeller University; Michaela Gack at the University of Chicago; So Young Kim at the Duke University Functional Genomics Core; Michael Gale, Jr., at the University of Washington; Adolfo García-Sastre at Mount Sinai School of Medicine; Shelton Bradrick and Mariano Garcia-Blanco at the University of Texas Medical Branch; and Sun Hur at Harvard University.

This study was supported by funds from the National Institutes of Health (K22AI100935 [S.M.H.] and R21Al124100 [S.M.H.]). Additional funding sources include the Burroughs Wellcome Fund (S.M.H.), a Duke Bridge Award (S.M.H.), and the Ford Foundation (C.V.).

The authors declare that they have no conflicts of interest with the contents of this article. The content is solely the responsibility of the authors and does not necessarily represent the official views of the National Institutes of Health.

\section{REFERENCES}

1. World Health Organization. 2017. Global hepatitis report. World Health Organization, Geneva, Switzerland. License: CC BY-NC-SA 3.0 IGO.

2. Grebely J, Hajarizadeh B, Dore GJ. 2017. Direct-acting antiviral agents for HCV infection affecting people who inject drugs. Nat Rev Gastroenterol Hepatol 14:641-651. https://doi.org/10.1038/nrgastro.2017.106.

3. Bartenschlager R, Baumert TF, Bukh J, Houghton M, Lemon SM, Lindenbach BD, Lohmann V, Moradpour D, Pietschmann T, Rice CM, Thimme R, Wakita T. 2018. Critical challenges and emerging opportunities in hepatitis $C$ virus research in an era of potent antiviral therapy: considerations for scientists and funding agencies. Virus Res 248:53-62. https://doi.org/ 10.1016/j.virusres.2018.02.016.

4. Horner SM, Gale M, Jr. 2013. Regulation of hepatic innate immunity by hepatitis C virus. Nat Med 19:879-888. https://doi.org/10.1038/nm.3253.

5. Brass V, Berke JM, Montserret R, Blum HE, Penin F, Moradpour D. 2008. Structural determinants for membrane association and dynamic organization of the hepatitis C virus NS3-4A complex. Proc Natl Acad Sci U S A 105:14545-14550. https://doi.org/10.1073/pnas.0807298105.
6. Wolk B, Sansonno D, Krausslich HG, Dammacco F, Rice CM, Blum HE Moradpour D. 2000. Subcellular localization, stability, and trans-cleavage competence of the hepatitis C virus NS3-NS4A complex expressed in tetracycline-regulated cell lines. J Virol 74:2293-2304. https://doi.org/10 .1128/jvi.74.5.2293-2304.2000.

7. Kohlway A, Pirakitikulr N, Barrera FN, Potapova O, Engelman DM, Pyle AM, Lindenbach BD. 2014. Hepatitis C virus RNA replication and virus particle assembly require specific dimerization of the NS4A protein transmembrane domain. J Virol 88:628-642. https://doi.org/10.1128/JVI.02052-13.

8. Morikawa K, Lange CM, Gouttenoire J, Meylan E, Brass V, Penin F, Moradpour D. 2011. Nonstructural protein 3-4A: the Swiss army knife of hepatitis C virus. J Viral Hepat 18:305-315. https://doi.org/10.1111/j.1365 -2893.2011.01451.x.

9. Roder AE, Vazquez C, Horner SM. 2019. The acidic domain of the hepatitis $C$ virus NS4A protein is required for viral assembly and envelopment through interactions with the viral E1 glycoprotein. PLoS Pathog 15:e1007163. https://doi.org/10.1371/journal.ppat.1007163. 
10. Li XD, Sun L, Seth RB, Pineda G, Chen ZJ. 2005. Hepatitis C virus protease NS3/4A cleaves mitochondrial antiviral signaling protein off the mitochondria to evade innate immunity. Proc Natl Acad Sci U S A 102: 17717-17722. https://doi.org/10.1073/pnas.0508531102.

11. Moradpour D, Penin F, Rice CM. 2007. Replication of hepatitis C virus. Nat Rev Microbiol 5:453-463. https://doi.org/10.1038/nrmicro1645.

12. Loo YM, Owen DM, Li K, Erickson AK, Johnson CL, Fish PM, Carney DS, Wang $T$, Ishida $\mathrm{H}$, Yoneyama M, Fujita T, Saito T, Lee WM, Hagedorn $\mathrm{CH}$, Lau DT, Weinman SA, Lemon SM, Gale M, Jr. 2006. Viral and therapeutic control of IFN-beta promoter stimulator 1 during hepatitis $C$ virus infection. Proc Natl Acad Sci U S A 103:6001-6006. https://doi.org/10.1073/ pnas.0601523103.

13. Horner SM, Liu HM, Park HS, Briley J, Gale M, Jr. 2011. Mitochondrialassociated endoplasmic reticulum membranes (MAM) form innate immune synapses and are targeted by hepatitis $C$ virus. Proc Natl Acad Sci U S A 108:14590-14595. https://doi.org/10.1073/pnas.1110133108.

14. Mottola G, Cardinali G, Ceccacci A, Trozzi C, Bartholomew L, Torrisi MR, Pedrazzini E, Bonatti S, Migliaccio G. 2002. Hepatitis C virus nonstructural proteins are localized in a modified endoplasmic reticulum of cells expressing viral subgenomic replicons. Virology 293:31-43. https://doi.org/10 .1006/viro.2001.1229.

15. Sumpter R, Jr, Loo YM, Foy E, Li K, Yoneyama M, Fujita T, Lemon SM, Gale M, Jr. 2005. Regulating intracellular antiviral defense and permissiveness to hepatitis $C$ virus RNA replication through a cellular RNA helicase, RIG-I. J Virol 79:2689-2699. https://doi.org/10.1128/JVI.79.5.2689-2699.2005.

16. Saito T, Hirai R, Loo YM, Owen D, Johnson CL, Sinha SC, Akira S, Fujita T, Gale M, Jr. 2007. Regulation of innate antiviral defenses through a shared repressor domain in RIG-I and LGP2. Proc Natl Acad Sci U S A 104:582-587. https://doi.org/10.1073/pnas.0606699104.

17. Israelow B, Narbus CM, Sourisseau M, Evans MJ. 2014. HepG2 cells mount an effective antiviral interferon-lambda based innate immune response to hepatitis C virus infection. Hepatology 60:1170-1179. https://doi.org/ 10.1002/hep.27227.

18. Oshiumi H, Matsumoto M, Hatakeyama S, Seya T. 2009. Riplet/RNF135, a RING finger protein, ubiquitinates RIG-I to promote interferon-beta induction during the early phase of viral infection. J Biol Chem 284: 807-817. https://doi.org/10.1074/jbc.M804259200.

19. Oshiumi H, Miyashita M, Matsumoto M, Seya T. 2013. A distinct role of Riplet-mediated K63-Linked polyubiquitination of the RIG-I repressor domain in human antiviral innate immune responses. PLoS Pathog 9:e1003533. https://doi.org/10.1371/journal.ppat.1003533.

20. Cadena C, Ahmad S, Xavier A, Willemsen J, Park S, Park JW, Oh SW, Fujita T, Hou F, Binder M, Hur S. 2019. Ubiquitin-dependent and -independent roles of E3 ligase Riplet in innate immunity. Cell 177:1187. https://doi .org/10.1016/j.cell.2019.03.017.

21. Gack MU, Shin YC, Joo CH, Urano T, Liang C, Sun L, Takeuchi O, Akira S, Chen Z, Inoue S, Jung JU. 2007. TRIM25 RING-finger E3 ubiquitin ligase is essential for RIG-I-mediated antiviral activity. Nature 446:916-920. https://doi.org/10.1038/nature05732.

22. Wang N, Liang Y, Devaraj S, Wang J, Lemon SM, Li K. 2009. Toll-like receptor 3 mediates establishment of an antiviral state against hepatitis C virus in hepatoma cells. J Virol 83:9824-9834. https://doi.org/10.1128/ JVI.01125-09.

23. Bellecave P, Sarasin-Filipowicz M, Donze O, Kennel A, Gouttenoire J, Meylan E, Terracciano L, Tschopp J, Sarrazin C, Berg T, Moradpour D, Heim MH. 2010. Cleavage of mitochondrial antiviral signaling protein in the liver of patients with chronic hepatitis $C$ correlates with a reduced activation of the endogenous interferon system. Hepatology 51: 1127-1136. https://doi.org/10.1002/hep.23426.

24. Meylan E, Curran J, Hofmann K, Moradpour D, Binder M, Bartenschlager R, Tschopp J. 2005. Cardif is an adaptor protein in the RIG-I antiviral pathway and is targeted by hepatitis C virus. Nature 437:1167-1172. https://doi.org/10.1038/nature04193.

25. Li K, Foy E, Ferreon JC, Nakamura M, Ferreon AC, Ikeda M, Ray SC, Gale $M$, Jr, Lemon SM. 2005. Immune evasion by hepatitis $C$ virus NS3/4A protease-mediated cleavage of the Toll-like receptor 3 adaptor protein TRIF. Proc Natl Acad Sci U S A 102:2992-2997. https://doi.org/10.1073/ pnas.0408824102.

26. Foy E, Li K, Wang C, Sumpter R, Jr, Ikeda M, Lemon SM, Gale M, Jr. 2003. Regulation of interferon regulatory factor-3 by the hepatitis $C$ virus serine protease. Science 300:1145-1148. https://doi.org/10.1126/science .1082604 .

27. Vazquez C, Tan CY, Horner SM. 2019. Hepatitis C virus infection is inhibited by a noncanonical antiviral signaling pathway targeted by NS3-NS4A. bioRxiv https://doi.org/10.1101/625640.

28. Kuiken C, Yusim K, Boykin L, Richardson R. 2005. The Los Alamos hepatitis $C$ sequence database. Bioinformatics 21:379-384. https://doi .org/10.1093/bioinformatics/bth485.

29. Braun P, von Heijne G. 1999. The aromatic residues Trp and Phe have different effects on the positioning of a transmembrane helix in the microsomal membrane. Biochemistry 38:9778-9782. https://doi.org/10 $.1021 /$ bi990923a.

30. de Planque MR, Bonev BB, Demmers JA, Greathouse DV, Koeppe RE, 2nd, Separovic F, Watts A, Killian JA. 2003. Interfacial anchor properties of tryptophan residues in transmembrane peptides can dominate over hydrophobic matching effects in peptide-lipid interactions. Biochemistry 42:5341-5348. https://doi.org/10.1021/bi027000r.

31. Wimley WC, White SH. 1996. Experimentally determined hydrophobicity scale for proteins at membrane interfaces. Nat Struct Biol 3:842-848. https://doi.org/10.1038/nsb1096-842.

32. Aligeti M, Roder A, Horner SM. 2015. Cooperation between the hepatitis $C$ virus $\mathrm{p7}$ and NS5B proteins enhances virion infectivity. J Virol 89: 11523-11533. https://doi.org/10.1128/JVI.01185-15.

33. Saito T, Owen DM, Jiang F, Marcotrigiano J, Gale M, Jr. 2008. Innate immunity induced by composition-dependent RIG-I recognition of hepatitis C virus RNA. Nature 454:523-527. https://doi.org/10.1038/nature07106.

34. Honda K, Takaoka A, Taniguchi T. 2006. Type I interferon [corrected] gene induction by the interferon regulatory factor family of transcription factors. Immunity 25:349-360. https://doi.org/10.1016/j.immuni.2006.08 009 .

35. Rustagi A, Gale M, Jr. 2014. Innate antiviral immune signaling, viral evasion and modulation by HIV-1. J Mol Biol 426:1161-1177. https://doi .org/10.1016/j.jmb.2013.12.003.

36. Foy E, Li K, Sumpter R, Jr, Loo YM, Johnson CL, Wang C, Fish PM, Yoneyama M, Fujita T, Lemon SM, Gale M, Jr. 2005. Control of antiviral defenses through hepatitis $C$ virus disruption of retinoic acid-inducible gene-I signaling. Proc Natl Acad Sci U S A 102:2986-2991. https://doi .org/10.1073/pnas.0408707102.

37. Clark K, Plater L, Peggie M, Cohen P. 2009. Use of the pharmacological inhibitor BX795 to study the regulation and physiological roles of TBK1 and $I_{\kappa} B$ kinase $\varepsilon$ : a distinct upstream kinase mediates Ser-172 phosphorylation and activation. J Biol Chem 284:14136-14146. https://doi.org/10 .1074/jbc.M109.000414.

38. Li K, Chen Z, Kato N, Gale M, Jr, Lemon SM. 2005. Distinct poly(I-C) and virus-activated signaling pathways leading to interferon-beta production in hepatocytes. J Biol Chem 280:16739-16747. https://doi.org/10 .1074/jbc.M414139200.

39. Gao D, Yang YK, Wang RP, Zhou X, Diao FC, Li MD, Zhai ZH, Jiang ZF, Chen DY. 2009. REUL is a novel E3 ubiquitin ligase and stimulator of retinoic-acid-inducible gene-I. PLoS One 4:e5760. https://doi.org/10 .1371/journal.pone.0005760.

40. Aguirre S, Luthra P, Sanchez-Aparicio MT, Maestre AM, Patel J, Lamothe F, Fredericks AC, Tripathi S, Zhu T, Pintado-Silva J, Webb LG, BernalRubio D, Solovyov A, Greenbaum B, Simon V, Basler CF, Mulder LC, Garcia-Sastre A, Fernandez-Sesma A. 2017. Dengue virus NS2B protein targets CGAS for degradation and prevents mitochondrial DNA sensing during infection. PLoS Pathog 2:17037.

41. Rajsbaum R, Albrecht RA, Wang MK, Maharaj NP, Versteeg GA, NistalVillán E, García-Sastre A, Gack MU. 2012. Species-specific inhibition of RIG-I ubiquitination and IFN induction by the influenza A virus NS1 protein. PLoS Pathog 8:e1003059. https://doi.org/10.1371/journal.ppat .1003059 .

42. Kato $H$, Takeuchi $O$, Sato $S$, Yoneyama $M$, Yamamoto $M$, Matsui $K$, Uematsu S, Jung A, Kawai T, Ishii KJ, Yamaguchi O, Otsu K, Tsujimura T, Koh CS, Reis e Sousa C, Matsuura Y, Fujita T, Akira S. 2006. Differential roles of MDA5 and RIG-I helicases in the recognition of RNA viruses. Nature 441:101-105. https://doi.org/10.1038/nature04734.

43. Fitzgerald KA, McWhirter SM, Faia KL, Rowe DC, Latz E, Golenbock DT, Coyle AJ, Liao SM, Maniatis T. 2003. IKKe and TBK1 are essential components of the IRF3 signaling pathway. Nat Immunol 4:491-496. https:// doi.org/10.1038/ni921.

44. Sharma S, tenOever BR, Grandvaux N, Zhou GP, Lin R, Hiscott J. 2003. Triggering the interferon antiviral response through an IKK-related pathway. Science 300:1148-1151. https://doi.org/10.1126/science.1081315.

45. Johnson CL, Owen DM, Gale M, Jr. 2007. Functional and therapeutic analysis of hepatitis C virus NS3.4A protease control of antiviral immune 
defense. J Biol Chem 282:10792-10803. https://doi.org/10.1074/jbc .M610361200.

46. Sumpter R, Jr, Wang C, Foy E, Loo YM, Gale M, Jr. 2004. Viral evolution and interferon resistance of hepatitis $C$ virus RNA replication in a cell culture model. J Virol 78:11591-11604. https://doi.org/10.1128/JVI.78.21 .11591-11604.2004.

47. Mali P, Yang L, Esvelt KM, Aach J, Guell M, DiCarlo JE, Norville JE, Church GM. 2013. RNA-guided human genome engineering via Cas9. Science 339:823-826. https://doi.org/10.1126/science.1232033.

48. Fredericksen B, Akkaraju GR, Foy E, Wang C, Pflugheber J, Chen ZJ, Gale $M, J r$. 2002. Activation of the interferon-beta promoter during hepatitis C virus RNA replication. Viral Immunol 15:29-40. https://doi.org/10 $.1089 / 088282402317340215$.

49. Yoneyama $M$, Kikuchi $M$, Natsukawa $T$, Shinobu N, Imaizumi T, Miyagishi M, Taira K, Akira S, Fujita T. 2004. The RNA helicase RIG-I has an essential function in double-stranded RNA-induced innate antiviral responses. Nat Immunol 5:730-737. https://doi.org/10.1038/ni1087.

50. Basler CF, Mikulasova A, Martinez-Sobrido L, Paragas J, Mühlberger E, Bray M, Klenk H-D, Palese P, García-Sastre A. 2003. The Ebola virus VP35 protein inhibits activation of interferon regulatory factor 3. J Virol 77: 7945-7956. https://doi.org/10.1128/jvi.77.14.7945-7956.2003.

51. Cong L, Ran FA, Cox D, Lin S, Barretto R, Habib N, Hsu PD, Wu X, Jiang W, Marraffini LA, Zhang F. 2013. Multiplex genome engineering using CRISPR/Cas systems. Science 339:819-823. https://doi.org/10.1126/ science. 1231143.

52. Kennedy EM, Whisnant AW, Kornepati AV, Marshall JB, Bogerd HP, Cullen
BR. 2015. Production of functional small interfering RNAs by an aminoterminal deletion mutant of human Dicer. Proc Natl Acad Sci U S A 112:E6945-E6954. https://doi.org/10.1073/pnas.1513421112.

53. Pfisterer U, Kirkeby A, Torper O, Wood J, Nelander J, Dufour A, Bjorklund A, Lindvall O, Jakobsson J, Parmar M. 2011. Direct conversion of human fibroblasts to dopaminergic neurons. Proc Natl Acad Sci U S A 108: 10343-10348. https://doi.org/10.1073/pnas.1105135108.

54. Hiscott J. 2007. Triggering the innate antiviral response through IRF-3 activation. J Biol Chem 282:15325-15329. https://doi.org/10.1074/jbc .R700002200.

55. Shalem O, Sanjana NE, Hartenian E, Shi X, Scott DA, Mikkelson T, Heckl D, Ebert BL, Root DE, Doench JG, Zhang F. 2014. Genome-scale CRISPRCas9 knockout screening in human cells. Science 343:84-87. https://doi .org/10.1126/science.1247005.

56. Heigwer F, Kerr G, Boutros M. 2014. E-CRISP: fast CRISPR target site identification. Nat Methods 11:122-123. https://doi.org/10.1038/nmeth .2812.

57. Schindelin J, Arganda-Carreras I, Frise E, Kaynig V, Longair M, Pietzsch T, Preibisch S, Rueden C, Saalfeld S, Schmid B, Tinevez JY, White DJ, Hartenstein V, Eliceiri K, Tomancak P, Cardona A. 2012. Fiji: an opensource platform for biological-image analysis. Nat Methods 9:676-682. https://doi.org/10.1038/nmeth.2019.

58. Aizaki H, Lee KJ, Sung VM, Ishiko H, Lai MM. 2004. Characterization of the hepatitis C virus RNA replication complex associated with lipid rafts. Virology 324:450-461. https://doi.org/10.1016/j.virol.2004.03.034. 